\section{(1)}

CrossMark

\title{
Fibrillin-2 is a key mediator of smooth muscle extracellular matrix homeostasis during mouse tracheal tubulogenesis
}

\author{
Wenguang Yin ${ }^{1,8}$, Hyun-Taek Kim¹, ShengPeng Wang ${ }^{2,3}$, Felix Gunawan ${ }^{1}$, \\ Rui $\mathrm{Li}^{2}$, Carmen Buettner ${ }^{1}$, Beate Grohmann ${ }^{1}$, Gerhard Sengle ${ }^{4,5}$, \\ Debora Sinner $^{6}$, Stefan Offermanns ${ }^{2,7}$ and Didier Y.R. Stainier ${ }^{1,8}$
}

Affiliations: ${ }^{1}$ Max Planck Institute for Heart and Lung Research, Dept of Developmental Genetics, Bad Nauheim, Germany. ${ }^{2}$ Max Planck Institute for Heart and Lung Research, Dept of Pharmacology, Bad Nauheim, Germany. ${ }^{3}$ Cardiovascular Research Center, School of Basic Medical Sciences, Xi'an Jiaotong University, Xi'an, China. ${ }^{4}$ Center for Biochemistry, Medical Faculty, University of Cologne, Cologne, Germany. ${ }^{5}$ Center for Molecular Medicine Cologne (CMMC), Cologne, Germany. ${ }^{6}$ Division of Neonatology and Pulmonary Biology, CCHMC, University of Cincinnati, College of Medicine Cincinnati, OH, USA. ${ }^{7}$ Center for Molecular Medicine, Goethe University, Frankfurt, Germany. ${ }^{8}$ W. Yin and D.Y.R. Stainier are joint senior authors.

Correspondence: Didier Y.R. Stainier, Dept of Developmental Genetics, Max Planck Institute for Heart and Lung Research, Ludwigstrasse 43, 61231 Bad Nauheim, Germany. E-mail: didier.stainierampi-bn.mpg.de

@ERSpublications

Defects in extracellular matrix formation lead to altered airway smooth muscle organisation in tracheal stenosis, and pharmacological decrease of p38 phosphorylation or matrix metalloproteinase activity partially attenuates these defects http://ow.ly/4zku30mWub3

Cite this article as: Yin W, Kim H-T, Wang SP, et al. Fibrillin-2 is a key mediator of smooth muscle extracellular matrix homeostasis during mouse tracheal tubulogenesis. Eur Respir J 2019; 53: 1800840 [https://doi.org/10.1183/13993003.00840-2018].

ABSTRACT Epithelial tubes, comprised of polarised epithelial cells around a lumen, are crucial for organ function. However, the molecular mechanisms underlying tube formation remain largely unknown. Here, we report on the function of fibrillin $(\mathrm{FBN}) 2$, an extracellular matrix (ECM) glycoprotein, as a critical regulator of tracheal tube formation.

We performed a large-scale forward genetic screen in mouse to identify regulators of respiratory organ development and disease. We identified Fbn2 mutants which exhibit shorter and narrowed tracheas as well as defects in tracheal smooth muscle cell alignment and polarity.

We found that FBN2 is essential for elastic fibre formation and Fibronectin accumulation around tracheal smooth muscle cells. These processes appear to be regulated at least in part through inhibition of p38-mediated upregulation of matrix metalloproteinases (MMPs), as pharmacological decrease of p38 phosphorylation or MMP activity partially attenuated the Fbn2 mutant tracheal phenotypes. Analysis of human tracheal tissues indicates that a decrease in ECM proteins, including FBN2 and Fibronectin, is associated with tracheomalacia.

Our findings provide novel insights into the role of ECM homeostasis in mesenchymal cell polarisation during tracheal tubulogenesis.

Published online March 07, 2019; republished March 15, 2019 to correct an error made in reproducing the Western blot image in figure $6 \mathrm{~b}$.

This article has supplementary material available from erj.ersjournals.com

Received: May 082018 | Accepted after revision: Nov 282018

Copyright OERS 2019 


\section{Introduction}

Smooth muscle cells surround epithelial and endothelial tubes in a number of organ systems, including the digestive tract, exocrine glands, lungs, kidneys and vasculature [1,2]. Recent data indicate that smooth muscle cells are required for tubulogenesis in lungs, gut and blood vessels [3-5]. The trachea is a stereotypical epithelial tube that is dorsally surrounded by smooth muscle which functions to provide elasticity and control tracheal contraction [6]. The tracheal epithelium originates from ventral foregut endoderm and is specified between embryonic days (E)8.25 and E9.0, followed by separation from the dorsal oesophagus after E9.5 [7]. The tracheal smooth muscle and cartilage originate from the surrounding splanchnic mesoderm. Smooth muscle cells differentiate at E11.5 [6], while cartilage development initiates as early as E9 [8], showing condensation by E13.5 [9, 10] and differentiation from E13.5 to E15.5 [11]. Tracheal epithelial cells initiate their differentiation after the establishment of smooth muscle and cartilage [12-15]. In humans, tracheal tube formation defects have been reported to cause tracheostenosis or tracheomalacia, which are characterised by a narrowing of the tracheal lumen or a deficiency of the supporting cartilage, respectively, and may lead to respiratory distress and death $[16,17]$. Studies on the cellular and molecular mechanisms underlying tracheal tubulogenesis have mostly focused on the role of epithelial cells $[18,19]$ as well as the complex signalling between the epithelium and mesenchyme $[9,20,21]$. However, the roles of smooth muscle and cartilage during tracheal tube formation remain poorly understood.

Another poorly understood aspect of tube development is the role of the extracellular matrix (ECM) secreted by smooth muscle cells and chondrocytes. Recent data indicate that ECM cues control tube formation by mediating epithelial cell polarisation [22, 23] and rearrangement [24], or orienting intercellular tension [25]. Notably, before our study, the role of the ECM in smooth muscle cell alignment and polarity during tracheal tubulogenesis had not been addressed.

\section{Materials and methods \\ Experimental animals}

$30 \mathrm{C} 57 \mathrm{BL} / 6 \mathrm{~J}$ male mice treated with a $3 \times 100 \mathrm{mg} \cdot \mathrm{kg}^{-1}$ dose of ethylnitrosourea (ENU) [26] were obtained from Monica Justice (Baylor College of Medicine, Houston, TX, USA). After a period of 10 weeks for the recovery of fertility, the mutagenised G0 males were crossed with C57BL/6J female mice. G1 males were outcrossed with C57BL/6J females to generate G2 females. Four G2 females were backcrossed to their G1 father, and the resulting G3 postnatal day (P)0 pups were subject to tracheal and lung dissection and analysis. All breeding colonies were maintained under a 12-h light and 12-h dark cycle. All mouse husbandry was performed under standard conditions in accordance with institutional (Max Planck Society) and local ethics committee (Regierungspräsidium Darmstadt, Hessen, Germany). All animal experiments were done in compliance with ethical guidelines and approved protocols.

\section{Whole-exome sequencing analysis and genotyping}

Genomic DNA isolated from two wild-type (WT) and two mutant mice, using a standard protocol was captured using SureSelect Mouse All Exon kit V1 (Agilent, Santa Clara, CA, USA), and sequenced using HiSeq 2000 (Illumina, San Diego, CA, USA), with minimum average 50× target sequence coverage (BGI-Hong Kong). Sequence reads were aligned to the C57BL/6J mouse reference genome (mm10) and analysed using CLCBio Genomic Workbench (Qiagen, Hilden, Germany), and GATK software. To minimise false negatives, variant calls were set at $5 \times$ minimum coverage and $\geqslant 20 \%$ alternate reads. Sequence variants were annotated to single nucleotide polymorphisms (SNPs) from dbSNP (version 142) and filtered against dbSNP128. For genotyping Fbn2 $2^{\text {T2547A/T2547A }}$ mice, primers Fwd (5'-TCCAGTCATGTCGAGGGCTA-3') and Rev (5'-CCAGCTTCTGCTCTTCTGCT-3') were used to generate a $\sim 420$-bp PCR amplicon. PCR products were sequenced using the primer Fwd ( $5^{\prime}$-TCCAGTCATGTCGAGGGCTA-3') and mutations were analysed using Biosystems Sequence Scanner Software (version 2.0; Foster City, CA, USA). Fbn2 null mice have been described previously [27]. For genotyping Fbn2 null mice, PCR was performed as previously described [28].

\section{Alcian blue staining of cartilage}

For wholemount staining of tracheal cartilage, dissected tracheas were fixed in 95\% ethanol for $12 \mathrm{~h}$ followed by overnight staining with $0.03 \%$ alcian blue dissolved in $80 \%$ ethanol and $20 \%$ acetic acid. Samples were cleared in $2 \%$ potassium hydroxide $(\mathrm{KOH})$.

\section{Wholemount staining of the limbs}

To perform wholemount staining of E13.5 forelimbs and hindlimbs [29], samples were incubated in $70 \%$ ethanol overnight at $4^{\circ} \mathrm{C}$, in $95 \%$ ethanol for $1 \mathrm{~h}$ and in acetone overnight at room temperature. Samples were then stained in $0.03 \%$ alcian blue overnight, cleared by $1 \% \mathrm{KOH}$ for $12 \mathrm{~h}$ and incubated in $50 \%$ glycerol:50\% $1 \% \mathrm{KOH}$ solution for $48 \mathrm{~h}$ at room temperature to make tissues transparent. To perform 
wholemount staining of P6 forelimbs and hindlimbs, samples were immersed in hot water for $30 \mathrm{~s}$ at $65^{\circ} \mathrm{C}$ to remove all skin, incubated in 95\% ethanol overnight and placed in acetone for $12 \mathrm{~h}$ at room temperature. Samples were stained in $0.03 \%$ alcian blue overnight, washed twice in $70 \%$ ethanol and incubated in 95\% ethanol overnight. Samples were pre-cleared in $1 \% \mathrm{KOH}$ for $1 \mathrm{~h}$ and incubated in $0.005 \%$ alizarin red for $2 \mathrm{~h}$ at room temperature. Samples were kept in 50\% glycerol:50\% $1 \% \mathrm{KOH}$ solution for imaging.

\section{Primary culture of mouse tracheal chondrocytes}

Tracheas were isolated from E14.5 embryos. To isolate chondrocytes [30], each trachea was incubated in $10 \mathrm{~mL}$ of collagenase D solution at $0.5 \mathrm{mg} \cdot \mathrm{mL}^{-1}$ for $12 \mathrm{~h}$ at $37^{\circ} \mathrm{C}$. Samples were then passed through $2-\mathrm{mL}$ Pasteur pipettes to disperse any cell aggregates. The cell suspension was filtered through a sterile $40 \mu \mathrm{m}$ cell strainer over a $50-\mathrm{mL}$ tube and centrifuged for $10 \mathrm{~min}$ at $400 \times g$ at room temperature. The pellet was washed in $2 \mathrm{~mL}$ PBS. Chondrocytes from each trachea were seeded on a 24 -well cell culture plate and cultured in DMEM/F-12 medium containing $10 \%$ fetal bovine serum (FBS) at $37^{\circ} \mathrm{C}$ in a $5 \%$ carbon dioxide $\left(\mathrm{CO}_{2}\right)$ incubator for 9 days.

\section{Alcian blue staining of cultured tracheal chondrocytes}

Mouse tracheal chondrocytes in primary cultures were washed twice in PBS and fixed in $1 \mathrm{~mL}$ of $4 \%$ glutaraldehyde for $15 \mathrm{~min}$ at room temperature. Samples were washed twice in $2 \mathrm{~mL}$ of $0.1 \mathrm{M}$ hydrogen chloride $(\mathrm{HCl})$ followed by staining with $1 \%$ alcian blue dissolved in $0.1 \mathrm{M} \mathrm{HCl}$ for $30 \mathrm{~min}$. After three washes in $2 \mathrm{~mL}$ of $0.1 \mathrm{M} \mathrm{HCl}$, samples were dried and rehydrated with $1 \mathrm{~mL}$ of $0.1 \mathrm{M} \mathrm{HCl}$.

\section{Quantification of relative alcian blue area}

Alcian blue area was measured using ImageJ (http://rsbweb.nih.gov/ij/). Relative alcian blue area was calculated by the ratio of alcian blue area to total cell area, and WT was assigned as 1 .

\section{Tracheal tube length measurements}

Tracheal tube length was determined by measuring the distance between the first and last tracheal cartilage rings.

\section{Respiratory rate measurements}

Respiratory rates were determined by measuring the number of breaths per minute. Unexpectedly, $\mathrm{Fbn2} 2^{T 2547 \mathrm{~A} / \mathrm{T} 2547 \mathrm{~A}}$ mice exhibit WT-like respiratory rates at rest. It is possible that $F b n 2^{T 2547 \mathrm{~A} / \mathrm{T} 2547 \mathrm{~A}}$ mice would display difficulties in breathing during high-intensity exercise.

\section{Wholemount immunostaining}

Tracheas were isolated from E12.5 to E16.5 embryos, and P0 and P60 mice. Tracheas were then fixed in $4 \%$ paraformaldehyde overnight at $4^{\circ} \mathrm{C}$ and washed three times in PBS for $1 \mathrm{~h}$ each. Samples were incubated in 5\% FBS/PBS/0.5\% Triton X-100/3\% bovine serum albumin for $12 \mathrm{~h}$ and then in primary antibodies overnight at $4^{\circ} \mathrm{C}$. After six washes in $0.5 \%$ Triton X-100/PBS for $1 \mathrm{~h}$ each, samples were incubated in secondary antibodies overnight at $4^{\circ} \mathrm{C}$. Samples were washed six times in $0.5 \%$ Triton X-100/ PBS for $1 \mathrm{~h}$ each and mounted for imaging. To visualise smooth muscle cells and chondroblasts, tracheas were stained for $\alpha$-smooth muscle actin (SMA) and SOX9, respectively. To visualise the Golgi apparatus, tracheas were stained for GM130. To visualise elastic fibres and Fibronectin (FN), tracheas were stained for Tropoelastin and FN.

\section{Quantification of short elastic fibres}

The number of short elastic fibres was counted per field of view using ImageJ.

\section{Immunostaining of cryosections}

Tracheas and lungs were dissected in PBS, fixed in $4 \%$ paraformaldehyde overnight at $4^{\circ} \mathrm{C}$, incubated in $10 \%$ sucrose and $30 \%$ sucrose for $24 \mathrm{~h}$ each at $4^{\circ} \mathrm{C}$, mounted in optimal cutting temperature (OCT) embedding compound, and sectioned at $10 \mu \mathrm{m}$ thickness. Immunostaining was performed using standard protocols. Immunostaining for phospho-p38 and $\alpha$-SMA was performed with WT and mutant trachea sections on the same slide.

Quantification of SFTPC ${ }^{+}$cell ratio

Surfactant protein C (SFTPC) ${ }^{+}$and total cell number was counted per field of view using ImageJ. SFTPC cell ratio was calculated as $\mathrm{SFTPC}^{+}$cells/total cells. 
Quantification of the mitotic angle

The epithelial plane is marked by a white dashed line, and the axis of mitosis by a green dashed line. The angle between these two lines represents the mitotic angle and was measured using ImageJ.

\section{In situ hybridisation of cryosections}

Tracheas were dissected in PBS, fixed in $4 \%$ paraformaldehyde overnight at $4^{\circ} \mathrm{C}$, mounted in OCT embedding compound, and sectioned at $10 \mu \mathrm{m}$ thickness. To perform in situ hybridisation [31], cryosections were permeabilised in $5 \mu \mathrm{g} \cdot \mathrm{mL}^{-1}$ proteinase $\mathrm{K}$ (04693159001; Roche, Indianapolis, IN, USA) for $15 \mathrm{~min}$ at room temperature, followed by acetylation for $2 \mathrm{~min}$ and pre-incubation in hybridisation buffer for $3 \mathrm{~h}$ at $70^{\circ} \mathrm{C}$, incubated with digoxigenin (DIG)-labelled RNA antisense probes overnight at $70^{\circ} \mathrm{C}$, washed, incubated with alkaline phosphatase-conjugated anti-digoxigenin antibody (Roche) overnight at $4^{\circ} \mathrm{C}$, washed, and the signal was detected with NBT/BCIP staining solution (Roche).

\section{Western blotting}

Isolated P7 tracheas were lysed using RIPA buffer (9806; Cell Signaling Technology, Danvers, MA, USA) supplemented with protease and phosphatase inhibitors (5872; Cell Signaling Technology). Lysates were centrifuged at $10000 \times g$ for $10 \mathrm{~min}$, subjected to SDS-PAGE and transferred to nitrocellulose membranes. Membranes were probed with primary and horseradish peroxidase-conjugated secondary antibodies (Cell Signaling Technology) and were developed using an enhanced chemiluminescent detection system (Pierce, Rockford, IL, USA).

\section{Gelatin zymography}

Isolated $\mathrm{P} 7$ tracheas were put in a lysis buffer $(0.025 \mathrm{M}$ Tris- $\mathrm{HCl}, \mathrm{pH} 7.5,0.1 \mathrm{M}$ sodium chloride $(\mathrm{NaCl}))$, and 1\% v/v Nonidet P-40 supplemented with Protease Inhibitor Cocktail-EDTA Free (04693159001; Roche). Lysates were centrifuged at $16000 \times \mathrm{g}$ for $10 \mathrm{~min}$ at $4^{\circ} \mathrm{C}$. The supernatant was collected and protein concentration measured. Equal amounts of protein were loaded per lane of gelatin zymography gels. The gel was then incubated in renaturing solution for $30 \mathrm{~min}$ at room temperature, washed twice in $300 \mathrm{~mL}$ of distilled water $\left(\mathrm{dH}_{2} \mathrm{O}\right)$, incubated in developing buffer for $30 \mathrm{~min}$ and incubated in fresh developing buffer for another $16 \mathrm{~h}$. After staining with Coomassie Blue, the gel was destained for examining gelatinolytic activity.

\section{Quantification of Western blot signals}

Fibronectin, active matrix metalloproteinases (MMP)2, MMP9, total p38, phospho-p38 and glyceraldehyde 3-phosphate dehydrogenase (GAPDH) levels were quantified using ImageJ. Fibronectin, active MMP2, MMP9, total p38 and phospho-p38 levels were normalised to the values yielded by GAPDH. Phospho-p38 fold change was calculated by the ratio of phospho-p38/p38 and WT was assigned to 1.

\section{Quantitative reverse transcription PCR}

Total RNA was extracted using a miRNeasy Mini Kit (Qiagen). cDNA was synthesised using the Maxima First Strand cDNA Synthesis Kit (ThermoFisher Scientific, Waltham, MA, USA), according to manufacturer's instructions. Quantitative real-time PCR was performed using Eco Real-Time PCR System (Illumina) and Maxima SYBR Green/Fluorescein qPCR Master Mix (ThermoFisher Scientific). Mean Ct values are shown in supplementary table 1 . Actb was used as a reference gene. The following primers were used: $A c t b$ forward 5'-CGGCCAGGTCATCACTATTGGCAAC-3' and Actb reverse 5'-GCCACAGGATTCCATACCCAAGAAG-3'; Atf2 forward 5'-ACTCCAACGCCAACAAGATTC-3' and Atf2 reverse 5'-TCTACAACAGAAGGCTCCTCAAT-3'; Fos forward 5'-GGGGACAGCCTTTCCTA-3' and Fos reverse 5'-CTGTCACCGTGGGGATAAAG-3'; Jun forward 5'-ACGACCTTCTACGACGATGC-3' and Jun reverse 5'-CCAGGTTCAAGGTCATGCTC-3'; Fbn 1 forward $5^{\prime}$-ACGGCTTTACTGGACCCCA-3' and Fbn 1 reverse 5'-ACATCTGGTTGCTTACCACAG-3'; Fbn2 forward 5'-CCACTCCTATTGCTGCCCAG-3' and Fbn2 reverse 5'-TTGGGGCGGGAACAGAATC-3'; Fibronectin forward 5'-GGCATCGGGGAGTGGCACTG-3' and Fibronectin reverse 5'-ATTGGGCTGGCTGGGGGTCT-3'; Fgf10 forward 5'-CGGGACCAAGAATGAAGACT-3' and Fgf10 reverse 5'-AGTTGCTGTTGATGGCTTTG-3'; Hspb1 forward 5'-ATCCCCT GAGGGCACACTTA-3' and Hspb1 reverse 5'-GGAATGGTGATCTCCGCTGAC-3'; Mmp2 forward $5^{\prime}$-ACCCAGATGTGGCCAACTAC-3' and $M m p 2$ reverse $5^{\prime}$-TACTTTTAAGGCCCGAGCAA-3'; $M m p 9$ forward 5' $5^{\prime}$ TAAGGACGGCAAATTTGGTT-3' and $M m p 9$ reverse $5^{\prime}$-CTTTAGTGGTGCAGGCAGAG-3'; $N f k b 1$ forward 5'-CGTAACAGCAGGACCCAAGG-3' and Nfkb1 reverse 5'-CTGTCATCCGTGCTTCCAGT-3'; Nfkb2 forward 5'-TTCAGAGTTGGGAGTGTGCG-3' and Nfkb2 reverse 5'-TGGTTACATGCAGGACACCC-3'; Rela forward 5'-CGGATTCCGGGCAGTGAC-3' and Rela reverse 5'-GAGGGGAAACAGA TCGTCCA-3'. 


\section{GM6001 and SB203580 injections; in vitro treatments with SB203580 and methylamine}

hydrochloride

GM6001 and SB203580 were dissolved in 100\% dimethyl sulfoxide (DMSO) (stock) and diluted with 0.9\% saline. Methylamine hydrochloride was dissolved in double-distilled water $\left(\mathrm{ddH}_{2} \mathrm{O}\right)$ (stock). Plugged C57BL6/J females were treated daily with intraperitoneal injections of GM6001 (50 mg. $\left.\mathrm{kg}^{-1}\right)$ or SB203580 $\left(1 \mu \mathrm{M} \cdot \mathrm{kg}^{-1}\right)$ between E12.5 and E14.5. The vehicle used for control injections was DMSO in $0.9 \%$ saline solution. E15.5 embryos were harvested for trachea isolation. For in vitro treatment with SB203580, isolated tracheas were cultured in DMEM/F-12 medium containing 0.1\% DMSO or $1 \mu \mathrm{M}$ SB203580 at $37^{\circ} \mathrm{C}$ in a $5 \% \mathrm{CO}_{2}$ incubator for $20 \mathrm{~h}$. For in vitro treatment with methylamine hydrochloride, isolated tracheas were cultured in DMEM/F-12 medium containing $1.5 \% \mathrm{ddH}_{2} \mathrm{O}$ or $30 \mu \mathrm{M}$ methylamine hydrochloride at $37^{\circ} \mathrm{C}$ in a $5 \% \mathrm{CO}_{2}$ incubator for $25 \mathrm{~h}$.

\section{Tracheal phenotype analysis after treatments}

Evaluation of tracheal tube length, smooth muscle orientation, elastic fibre formation and Fibronectin accumulation after GM6001 or SB203580 injection or treatment was performed prior to genotyping.

\section{Antibodies}

The following antibodies were used: mouse anti- $\alpha$-SMA-Cy3 (1:1000, C6198; Sigma-Aldrich, St Louis, MO, USA); rat anti-CDH1 (1:500, sc-59778; Santa Cruz Biotechnology, Dallas, TX, USA); rabbit anti-SOX9 (1:400, AB5535; Millipore, Burlington, MA, USA); goat anti-SOX9 (1:200, AF3075; R\&D Systems, Minneapolis, MN, USA); sheep anti-GM130 (1:50, AF8199; R\&D Systems); rabbit anti-Ki67 (1:400, \#9027; Cell Signaling Technologies); mouse anti-PCNA (1:400, sc-56; Santa Cruz Biotechnology); rabbit antiCleaved Caspase-3 (1:600, \#9661; Cell Signaling Technologies); rabbit anti-Phospho-p38 (1:100 for immunostaining and 1:1000 for Western blots, \#9215; Cell Signaling Technologies); rabbit anti-p38 (1:1000, \#9212; Cell Signaling Technologies); rabbit anti-MMP-9 (1:1000, AB19016; Millipore); rabbit-MMP-2 (1:1000, AB19015; Millipore); rabbit anti-FBN2 (1:5000 for immunostaining and 1:2000 for Western blots); guinea-pig anti-FBN2 (1:500); rabbit anti-Tropoelastin (1:1000 for immunostaining and 1:1000 for Western blots, ab21600; Abcam, Cambridge, UK); sheep anti-Fibronectin (1:1000, AF1918; Novus Biologicals, Centennial, CO, USA); rabbit anti-Fibronectin (1:500 for immunostaining and 1:1000 for Western blots, F3648; Sigma); goat anti-Aggrecan (1:200, AF1220; R\&D Systems); rabbit anti-COL2A1 (1:50, AB761; Millipore); rabbit anti-PH3 (1:400, 06-570; Millipore); rat anti-RAGE (1:400, MAB1179; R\&D Systems); rat anti-PECAM (1:200, 553370; BD Biosciences, Franklin Lakes, NJ, USA); goat anti-CC10 (1:200, T-18; Santa Cruz); rabbit anti-SFTPC (1:400, AB3786; Millipore); mouse anti-acetylated $\alpha$-tubulin (1:2000, T-7451; Sigma); goat anti-Vimentin (1:200, C-20; Santa Cruz); and rabbit anti-GAPDH (1:3000, \#2118; Cell Signaling Technologies). Polyclonal rabbit and guinea-pig anti-Fibrillin-2 antisera were raised against the N-terminal recombinant human Fibrillin-2 polypeptide $\mathrm{rF} 86\left(\mathrm{Gln}^{29}-\mathrm{Asp}^{535}\right)$ in 293 Epstein-Barr virus nuclear antigen (EBNA) cells [32]. Antisera were purified before use by affinity chromatography on a column with antigen coupled to cyanogen bromide-activated Sepharose (GE Healthcare, Chicago, IL, USA).

\section{Explant culture of mouse embryonic tracheas and lungs, and in vitro treatment with protease}

Tracheas and lungs were isolated from E13.5 embryos and cultured using an established protocol [33]. For in vitro treatment with elastase or collagenase type II, isolated tracheas and lungs were cultured in DMEM/F-12 medium or DMEM/F-12 medium containing $2 \mathrm{mU} \cdot \mathrm{mL}^{-1}$ elastase (LS002290; Worthington Biochemical Corporation, Lakewood, NJ, USA) or DMEM/F-12 medium containing $2 \mathrm{mU} \cdot \mathrm{mL}^{-1}$ collagenase type II (9001-12-1; Gibco, Waltham, MA, USA) at $37^{\circ} \mathrm{C}$ in a $5 \% \mathrm{CO}_{2}$ incubator for $28 \mathrm{~h}$.

\section{Ex vivo trachea physiology}

$2 \mathrm{~mm}$ sections of tracheas were isolated from P1 pups and kept in Krebs solution $(119 \mathrm{mM} \mathrm{NaCl}, 4.7 \mathrm{mM}$ $\mathrm{KCl}, 2.5 \mathrm{mM} \mathrm{CaCl}_{2}, 1.17 \mathrm{mM} \mathrm{MgSO}_{4}, 20 \mathrm{mM} \mathrm{NaHCO}_{3}, 1.18 \mathrm{mM} \mathrm{KH}_{2} \mathrm{PO}_{4}, 0.027 \mathrm{mM}$ EDTA, $11 \mathrm{mM}$ glucose) aerated with carbogen at $37^{\circ} \mathrm{C}$. Tracheal rings were mounted in a wire-myograph system (610-M; Danish Myo Technology, Aarhus, Denmark) and a resting tension of $2 \mathrm{mN}$ was applied for each ring as a baseline. Contractile responses were determined by cumulative administration of indicated acetylcholine concentrations. For spontaneous contraction analysis of fetal tracheas, E13.5 tracheas were isolated and kept in PBS for time-lapse imaging for 10 minutes every 1 second with a Zeiss LSM 800 inverted laser scanning confocal microscope. The amplitude of spontaneous contractions was defined as " 1 - the ratio of tracheal smooth muscle minimum width after contraction to maximum width after relaxation".

\section{Human tracheomalacia samples}

Paraffin sections of deidentified human tracheomalacia samples and healthy tracheal tissues (controls) were provided by Cincinnati Children's Hospital Medical Center Biobank Core facility (Cincinatti, OH, USA). The clinical diagnosis of tracheomalacia was confirmed by histological analysis. The study protocol 
and tissue usage were approved by the institutional ethics committee. Written informed consent was obtained from all patients' families prior to collection of samples by "Better Outcomes for Children" biorepository.

\section{Imaging}

Imaging of wholemount tracheas, lungs and limbs, and trachea and lung sections was performed using a Nikon SMZ25, Nikon 90i, Zeiss Axio Imager.A2, Zeiss LSM 800 inverted or 880 upright laser scanning confocal microscope. Quantification of tube length, distance between the C-shaped rings, ring width, smooth muscle area, smooth muscle cell orientation and nuclear aspect ratio, Golgi apparatus position relative to the nucleus, elastic fibre orientation, short elastic fibres, immunofluorescence intensity, cell number and mean linear intercept was performed using ImageJ. In straight and bent tracheas, tube length was measured by drawing a freehand line along the outer edge of the trachea in an anterior-posterior direction in ImageJ.

\section{Statistical analysis}

Statistical analyses were performed using GraphPad software (San Diego, CA, USA). p-values were calculated using t-tests $\left({ }^{*}: \mathrm{p}<0.05 ;{ }^{* *}: \mathrm{p}<0.01 ;{ }^{* * *}: \mathrm{p}<0.001\right.$; Ns: nonsignificant).

\section{Results}

\section{Fbn2 $2^{\text {T2547A/T2547A }}$ mice exhibit tracheal tube formation defects}

To identify novel loci regulating mouse respiratory organ formation, we conducted a large-scale forward genetic screen using ENU mutagenesis. One of the recessive mutants identified in this screen exhibits lower body weight (figure $1 \mathrm{a}$ and $\mathrm{b}$ and supplementary figure S1a and b), decreased nose to anus length (supplementary figure S1a and c), a shortened trachea (figure $1 \mathrm{c}$ and $\mathrm{d}$ and supplementary figure S1d), fewer cartilage rings (figure $1 \mathrm{c}$ and e), a narrowed tracheal lumen (figure if and g), but WT-like respiratory rate (supplementary figure S1e). These mutant animals are born in the expected Mendelian ratio, indicating that this mutation does not cause embryonic lethality. To identify the phenotype-causing mutation, we performed whole-exome sequencing of G4 genomic DNA samples and identified Fbn2, which encodes an ECM glycoprotein, as a candidate gene (figure 1h). Next, we carried out genetic linkage analysis by genotyping 189 G4, G5 and G6 mutant animals and found complete linkage between the tracheal phenotypes and the Fbn2 $2^{T 2547 A / T 2547 A}$ allele (figure 1i). The identified allele carries a nonsense mutation (c.T2547A) at a highly conserved amino acid (figure $1 \mathrm{j}$ ), which is predicted to cause a truncated protein with 848 amino acids (p.C849X) (figure 1k). We then carried out a complementation test by crossing mice carrying the ENU-induced Fbn2 allele $\left(F b n 2^{T 2547 A /+}\right)$ with mice carrying a Fbn2 deletion allele [27], and found that complementation did not occur in the $F b n 2^{-/ T 2547 A}$ double-heterozygous animals (figure 11), indicating that loss of Fbn2 function is likely to be responsible for the observed tracheal phenotypes in the Fbn2 $2^{T 2547 A / T 2547 A}$ mice. To further test the role of Fbn2 in tracheal development, we analysed tracheal formation in $\mathrm{Fbn}^{-/-}$mice. $\mathrm{Fbn} 2^{-/-}$mice exhibit a shortened trachea with fewer and fractured cartilage rings (supplementary figure S2a-c) and disorganised smooth muscle (supplementary figure S2c and d). Fbn2 $2^{-/-}$mice have been reported to exhibit skeletal limb abnormalities [27]. We thus examined limb formation in Fbn2 $2^{T 2547 A / T 2547 A}$ mice. Interestingly, Fbn2 ${ }^{\text {T2547A/T2547A }}$ mice exhibit bilateral syndactyly of the central digits in the hindlimbs as early as E13.5 (supplementary figure S3a), and until adulthood (supplementary figure S3b and c). However, we observed no significant phenotype in $F b n 2^{T 2547 A / T 2547 A}$ forelimbs (supplementary figure S3d-f). To test for a possible role of Fbn2 in lung development, we analysed the intrapulmonary airways and the lungs. Fbn $2^{\text {T2547A/T2547A }}$ mice exhibit cartilage formation defects in the intrapulmonary airways (supplementary figure S4a-d). However, we observed no significant phenotype in lung branching morphogenesis (supplementary figure S5a and b), alveologenesis (supplementary figure S5c and d), or lung cell differentiation (supplementary figure S6a-i) in $F b n 2^{T 2547 A / T 2547 A}$ animals. In addition, we examined tracheal tube formation and limb patterning in Fbn $2^{T 2547 A /+}$ animals. Fbn2 $2^{T 2547 A /+}$ mice exhibit WT-like tracheal length (supplementary figure S7a and b), cartilage formation (supplementary figure S7a and c), smooth muscle cell orientation (supplementary figure S7d and e) and limb patterning (supplementary figure S7f), indicating haplosufficiency of Fbn2. Altogether, these findings strongly suggest that Fbn2 regulates tracheal tube formation.

\section{FBN2 surrounds tracheal smooth muscle cells and chondroblasts}

To examine the spatiotemporal expression patterns of FBN2 in the developing mouse trachea, we performed immunostaining during embryonic and postnatal stages using a polyclonal antibody raised against amino acids 29-535. FBN2 appears to localise around the tracheal mesenchyme including the smooth muscle cells and chondroblasts as early as E12.5 (figure 2a). Interestingly, FBN2 expression levels appear gradually reduced around chondroblasts during chondrocyte differentiation from E14.5 to E18.5 (figure 2b), such that FBN2 immunostaining was barely detectable around tracheal chondrocytes at 

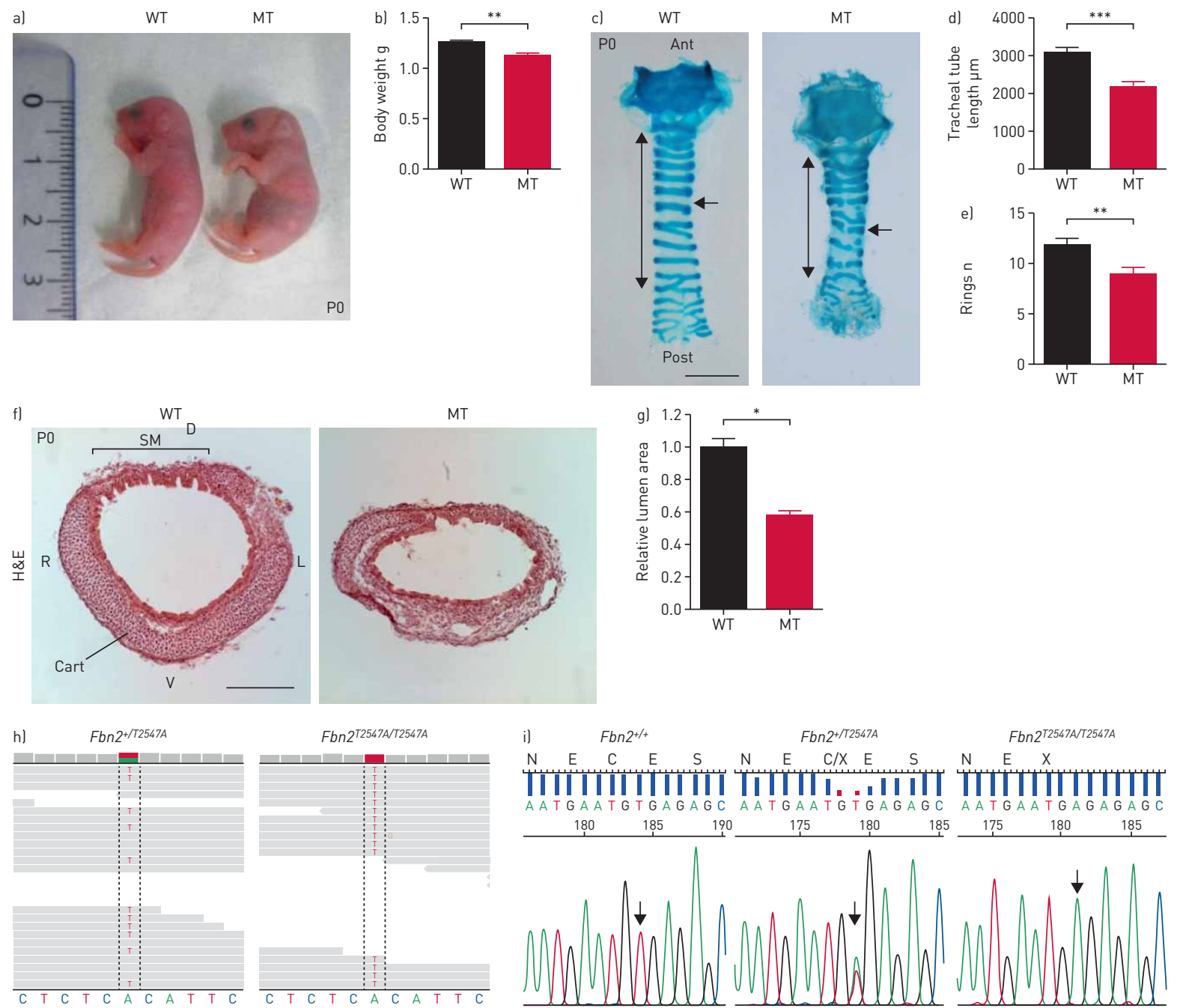

j)

Homosapiens NTPGSYSCTCPPGY VFRTETETCEDINECESNPCVNGAC Papio anubis NTPGSYSCTCPPGYVFRTETETCEDINECESNPCVNGAC Mus musculus NTPGSYSCTCPPGYVFRTETETCEDVNECESNPCVNGAC Danio rerio NTPGSYTCSCPKGFSFKADSETCEDINECDSNPC I NG I C

k)

WT FBN2 (2907 aa)
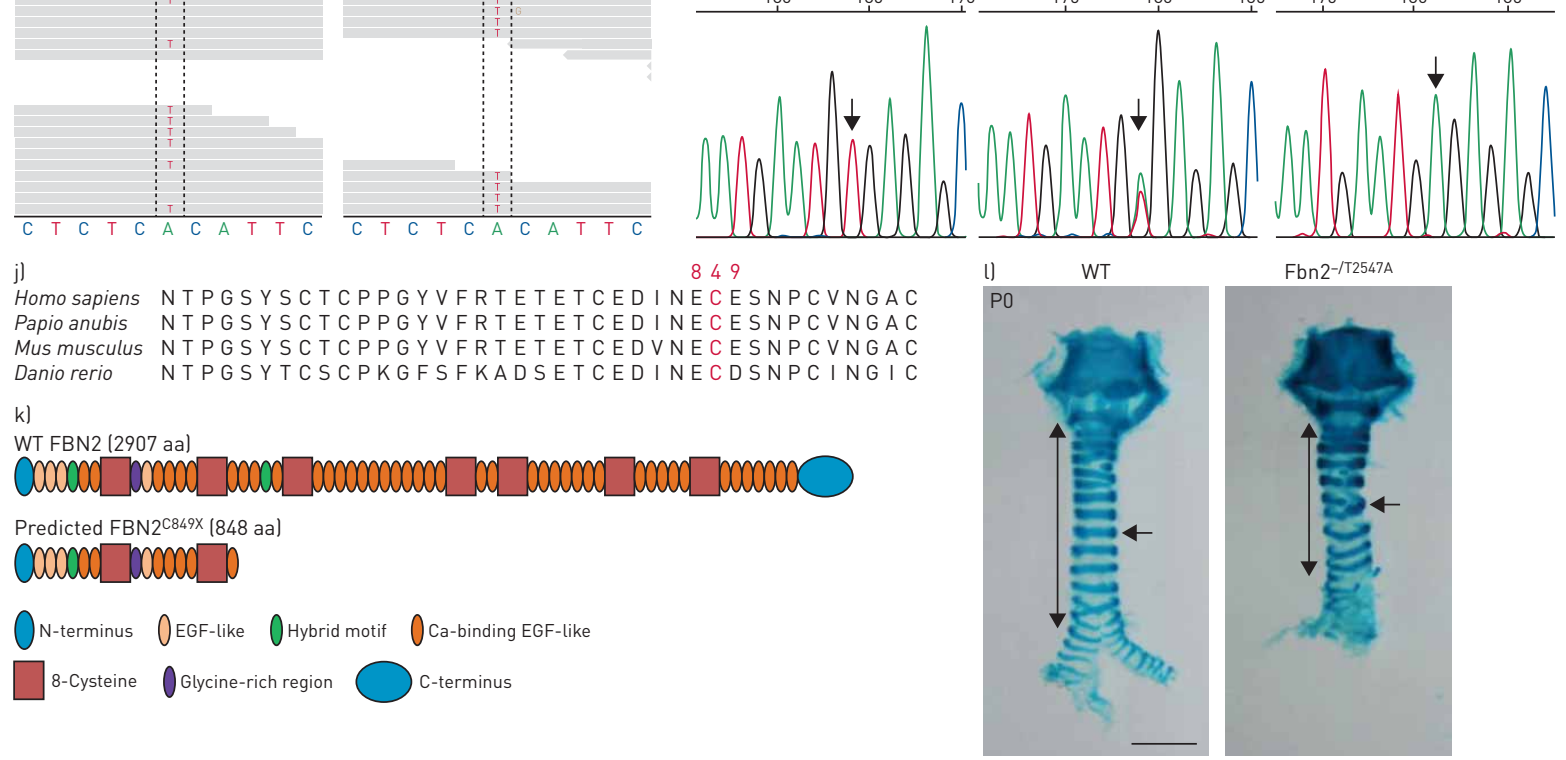

$\mathrm{Fbn2}-$-T2547A

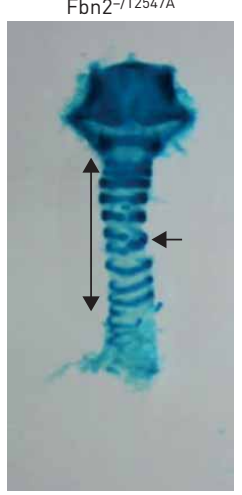

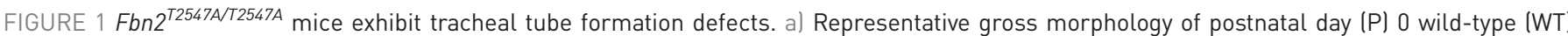
$(n=16)$ and mutants (MT) ( $n=16)$. b) Quantification of body weight of PO WT ( $n=15)$ and mutants ( $n=15)$. c) Representative images of ventral views of wholemount tracheas stained with alcian blue from PO WT $(n=24)$ and mutants $(n=24)$. Double-ended arrows indicate tracheal tube length. Arrows point to tracheal cartilage rings. Scale bar $=1 \mathrm{~mm}$. d) Quantification of PO WT ( $\mathrm{n}=15)$ and mutant ( $\mathrm{n}=15)$ tracheal tube length. e) Quantification of the number of tracheal cartilage rings from PO WT $(n=15)$ and mutants $(n=15)$. f) Representative images of transverse sections of tracheas stained with haematoxylin and eosin (H\&E) from PO WT ( $n=5)$ and mutants $(n=5)$. Scale bar $=200 \mu \mathrm{m}$. g) Quantification of tracheal lumen area from P0 WT $(n=5)$ and mutants $(n=5)$. h) Whole-exome sequencing of WT control (Fbn2 heterozygous, $n=2)$ and mutant (Fbn2 homozygous, $n=2)$ samples; green indicates the WT nucleotide A; red indicates the mutant nucleotide T. i) Sequence of WT and mutant genomic DNA around the lesion. DNA sequence chromatograms show TGT for cysteine in WT $(n=112)$ (left). TGT and TGA in heterozygous mutants, and TGA for the stop codon in homozygous mutants ( $n=124)$ (right). Arrows point to the mutation site. j) Evolutionary conservation of the p.C849 residue in vertebrates. k) Domain structure of WT Fibrillin (FBN)2 and FBN2 ${ }^{\mathrm{C} 849 \mathrm{X}}$. l) Representative images of ventral views of wholemount tracheas stained with alcian blue from PO WT $(n=15)$ and Fbn2 $2^{-T 2547 A}$ double heterozygous animals $(n=15)$. Double-ended arrows indicate tracheal tube length. Arrows point to tracheal cartilage rings. Scale bar=1 mm. Ant: anterior; Post: posterior; D: dorsal; V: ventral; R: right; L: left; Cart: cartilage; SM: smooth muscle; EGF: epidermal growth factor. Data are presented as mean \pm SD. ${ }^{*}: p<0.05 ;{ }^{* *}: p<0.01 ;{ }^{* * *}: p<0.001$ unpaired t-test. 

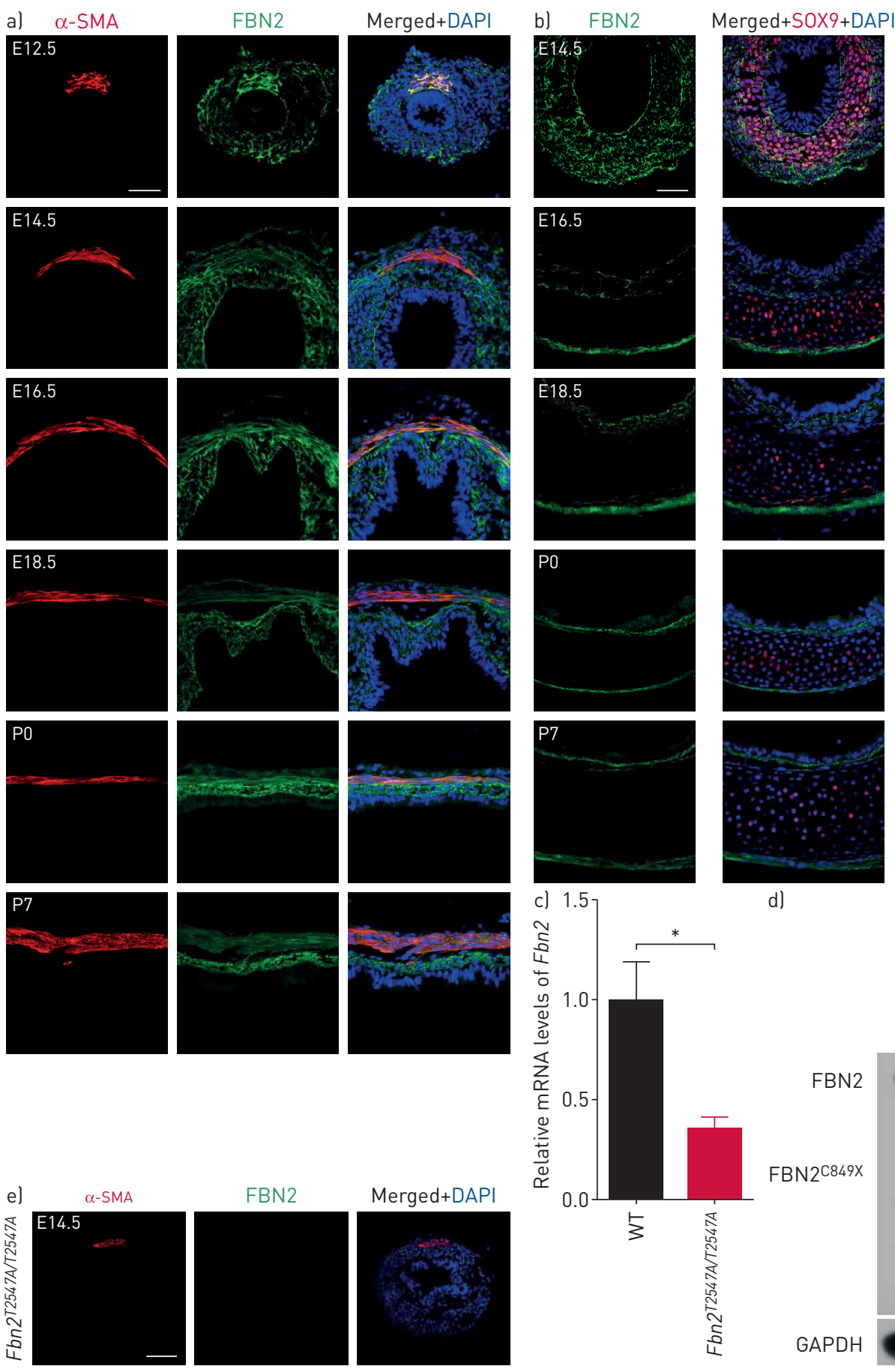

d)

FIGURE 2 Fibrillin (FBN)2 localises around the tracheal smooth muscle cells and chondroblasts. a) Immunostaining for FBN2 (green) and $\alpha$-smooth muscle actin (SMA) (red), and 4',6-diamidino-2-phenylindole (DAPI) staining (blue) of transverse sections of wild-type (WT) tracheas $(n=6)$ in the whole tracheal region (embryonic day (E)12.5) or mostly in smooth muscle regions at later stages. Scale bar=50 $\mu \mathrm{m}$. b) Immunostaining for FBN2 (green) and SOX9 (red), and DAPI staining (blue) of transverse sections of WT tracheas $(n=6)$ mostly in cartilage regions at several embryonic and postnatal stages. Scale bar $=50 \mu \mathrm{m}$. c) Quantitative reverse transcription PCR analysis of Fbn2 expression in E12.5 WT (n=4) and Fbn2 T2547A/T2547A $(n=4)$ tracheas. d) Western blotting for FBN2 and glyceraldehyde 3-phosphate dehydrogenase (GAPDH) in postnatal day (P)7 WT $(n=4)$ and Fbn2 ${ }^{T 2547 A / T 2547 A}(n=4)$ tracheas. e) Immunostaining for FBN2 (green) and $\alpha$-smooth muscle actin (SMA) (red), and DAPI staining (blue) of transverse sections of E14.5 Fbn2 T2547A/T2547A tracheas $(n=6)$. Scale bar $=100 \mu \mathrm{m}$. Data are presented as mean \pm SD. *: $p<0.05$; unpaired t-test. 
postnatal stages (figure 2b). We then examined Fbn2 mRNA expression in developing tracheas. In E14.5 tracheas, Fbn2 appeared to be exclusively expressed in mesenchymal cells, including the chondroblasts (supplementary figure S8a). However, at E16.5, Fbn2 mRNA levels appeared to be reduced in tracheal chondrocytes (supplementary figure S8b) and were mainly detected in other mesenchymal cells, including tracheal smooth muscle cells (supplementary figure S8c). These data indicate that FBN2 is required for tracheal smooth muscle cell and chondrocyte development at early embryonic stages. Next, we investigated Fbn2 $2^{T 2547 A / T 2547 A}$ mRNA and protein: mutant mRNA levels were significantly reduced compared to WT (figure $2 \mathrm{c}$ and supplementary table 1), suggesting mRNA decay. No FBN2 immunoblot (figure 2d) or immunostaining (figure 2e) signal was observed in the mutant animals, indicating that the truncated Fbn2 $2^{\text {T2547A/T2547A }}$ protein is not synthesised or is highly unstable. These results indicate that the $\mathrm{Fbn2} 2^{\mathrm{T2547A} / T 2547 \mathrm{~A}}$ mutation leads to a reduction of FBN2 at both the mRNA and protein levels.

Fbn2 $2^{\text {T2547A/T2547A }}$ mice display defects in tracheal elongation, cartilage ring formation and smooth muscle organisation

To examine the formation of the trachea in more detail, we performed a systematic analysis of tracheal tube development. Fbn2 $2^{T 2547 A / T 2547 A}$ mice display no obvious differences in tracheal tube length compared to WT siblings at E12.5 (figure 3a and c). However, starting at E13.5, we observed that Fbn2 ${ }^{T 2547 A / T 2547 A}$ tracheas were shorter than their WT counterparts (figure $3 a$ and $c$ ), and this phenotype became more pronounced at postnatal stages (figure $3 b$ and $c$ ). These results suggest that impaired tracheal tube elongation occurs after smooth muscle differentiation, which starts at E11.5 [6]. To identify alterations in tracheal architecture, we performed a structural analysis of the cartilage and smooth muscle. Cartilage formation occurs as a result of the condensation of mesenchymal cells followed by chondrogenic differentiation [34]. Starting at E13.5, chondrogenic nodules resulting from $\mathrm{SOX9}^{+}$mesenchymal cell condensation were observed (figure 3d). However, Fbn2 $2^{T 2547 A / T 2547 A}$ tracheas exhibited a reduced number of C-shaped rings starting at E13.5 and until adulthood (figure 3d-f). Moreover, starting at E14.5, an increased distance between the C-shaped rings (figure $3 \mathrm{~d}$ and $\mathrm{g}$ ) and a decreased ring width (figure $3 \mathrm{~d}$ and h) were observed in Fbn2 $2^{T 2547 A / T 2547 A}$ tracheas. FBN2 levels decline progressively during chondrogenic differentiation of mouse ATDC5 cells [35], similar to that which is observed in tracheal chondroblasts (figure $2 \mathrm{~b}$ ). Thus, we hypothesised that FBN2 deficiency might lead to compromised tracheal chondrocyte differentiation. To test this hypothesis, we examined expression levels of aggrecan (AGC1) and type II collagen (COL2A1), chondrocyte-specific markers [34], and observed that AGC1 (supplementary figure S9a and b) and COL2A1 (supplementary figure S9c and d) levels were significantly reduced in $\mathrm{Fbn2} 2^{\text {T2547A/T2547A }}$ tracheas. In contrast, we did not find obvious alterations in apoptosis (supplementary figure $\mathrm{S} 10 \mathrm{a}-\mathrm{c}$ ) or $\mathrm{SOX}^{+}$chondroblast proliferation (supplementary figure S10d and e) between WT and Fbn2 ${ }^{\text {T2547A/T2547A }}$ tracheas, indicating that FBN2 deficiency causes abnormal tracheal rings by inhibiting chondrocyte differentiation. We then isolated immature chondrocytes from E14.5 tracheas and cultured them for 9 days. Interestingly, $F b n 2^{T 2547 A / T 2547 A}$ chondrocytes exhibited reduced alcian blue staining in primary cultures (supplementary figure S11a and b), suggesting that Fbn2 regulates cartilage formation in a cell-autonomous manner. Since altered smooth muscle morphogenesis can affect tube elongation $[4,10,36]$, we analysed tracheal smooth muscle development. Smooth muscle cells, which are positioned dorsally in the trachea, displayed no obvious differences between WT and Fbn2 $2^{T 2547 A / T 2547 A}$ animals at E12.5 (figure $3 \mathrm{i}$ and $\mathrm{j}$ ), indicating that FBN2 is dispensable for smooth muscle differentiation and early steps in smooth muscle morphogenesis. Disorganised smooth muscle stripes of decreased area were first observed in $F b n 2^{T 2547 A / T 2547 A}$ tracheas at E13.5 (figure $3 \mathrm{i}$ and $\mathrm{j}$ ) and became more noticeable starting at E14.5 (figure $3 \mathrm{i}$ and $\mathrm{j}$ ). In contrast, Fbn2 ${ }^{T 2547 \mathrm{~A} / T 2547 \mathrm{~A}}$ tracheal smooth muscle cells did not exhibit significant changes in apoptosis (supplementary figure S10a-c) or proliferation (supplementary figure S10f and g) compared to WT. Similarly, mitotic spindle orientation (supplementary figure S12a and b) and cell proliferation (supplementary figure $\mathrm{S} 12 \mathrm{c}$ and $\mathrm{d}$ ) in the tracheal epithelium of Fbn2 $2^{\text {T2547A/T2547A }}$ mice appeared to be unaffected compared to WT. Since Fgf10 is expressed in tracheal smooth muscle cells and modulates airway patterning [20], we also tested Fgf10 mRNA levels. Fbn2 $2^{\text {T2547A/T2547A }}$ tracheas exhibited no significant difference in Fgf10 expression levels (supplementary figure S12e and supplementary table 1), indicating that Fbn2 signals via a Fgflo independent pathway to regulate tracheal patterning. Collectively, these results suggest that chondrocyte differentiation and smooth muscle organisation are essential for tracheal tube formation.

\section{Fbn2 mediates smooth muscle cell orientation and polarisation}

Longitudinal smooth muscle has been reported to restrict gut tube elongation by compressing the mesenchyme and attached epithelium [4]. Our findings that FBN2 is required for smooth muscle organisation led us to hypothesise that the tracheal elongation defects observed in Fbn2 $2^{\text {T2547A/T2547A }}$ mice might be due to altered smooth muscle cell alignment. Thus, we performed a systematic analysis of smooth muscle cell orientation. In WT animals, differentiated tracheal smooth muscle cells exhibit 

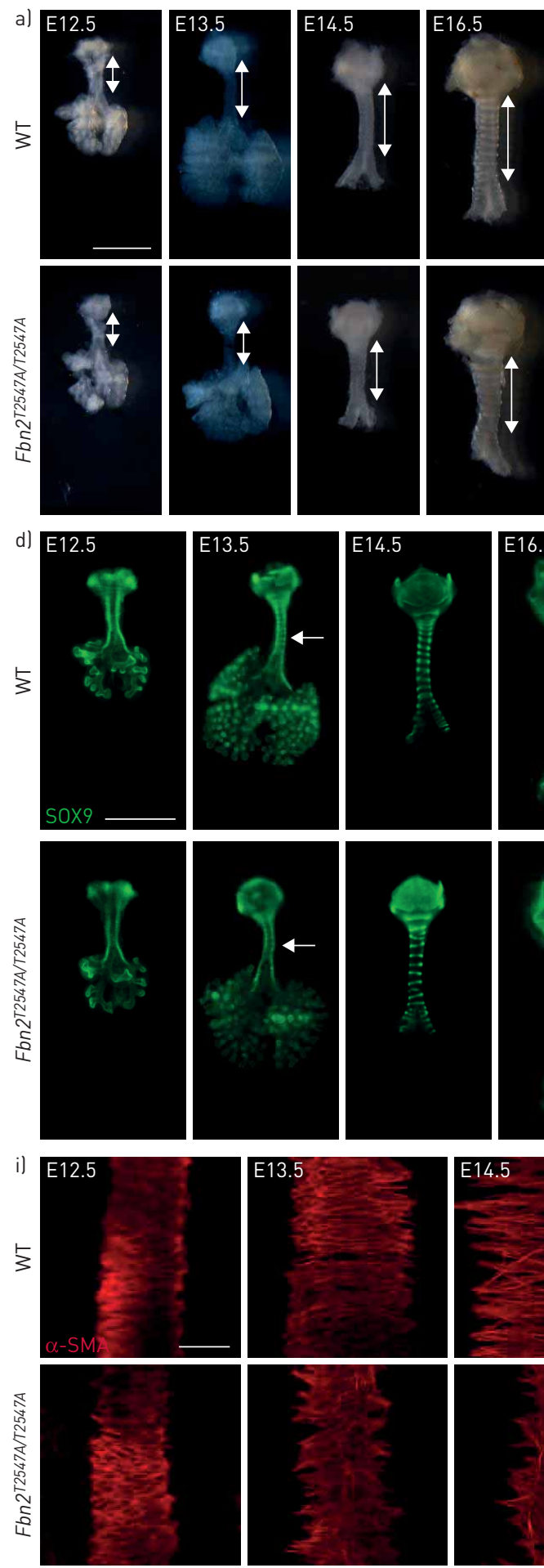
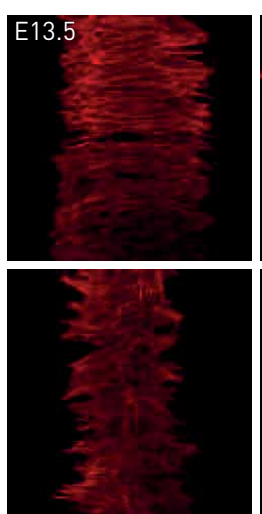
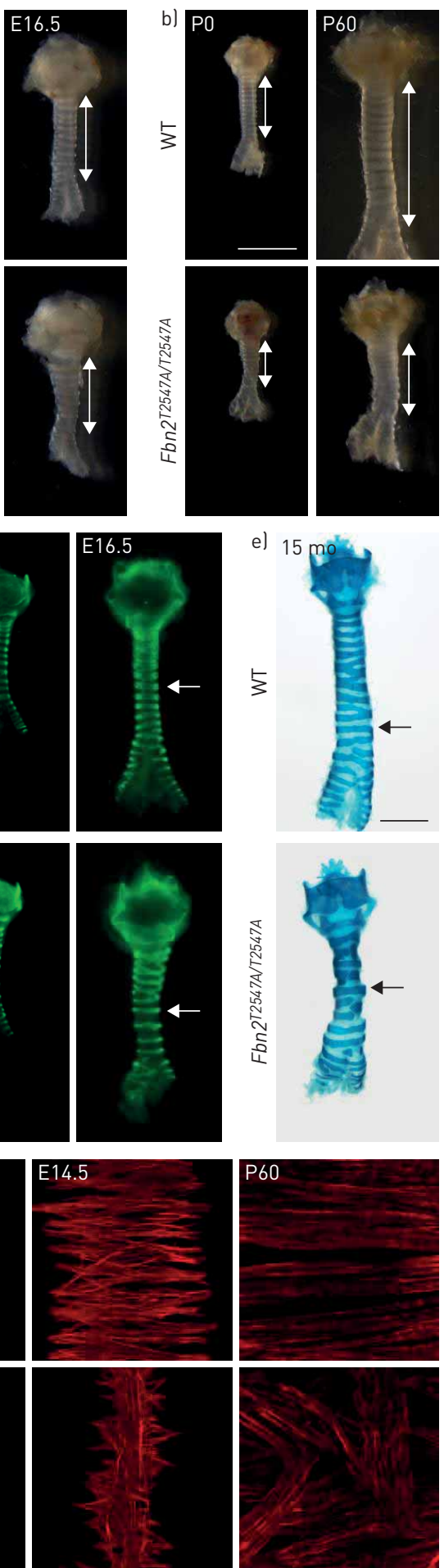
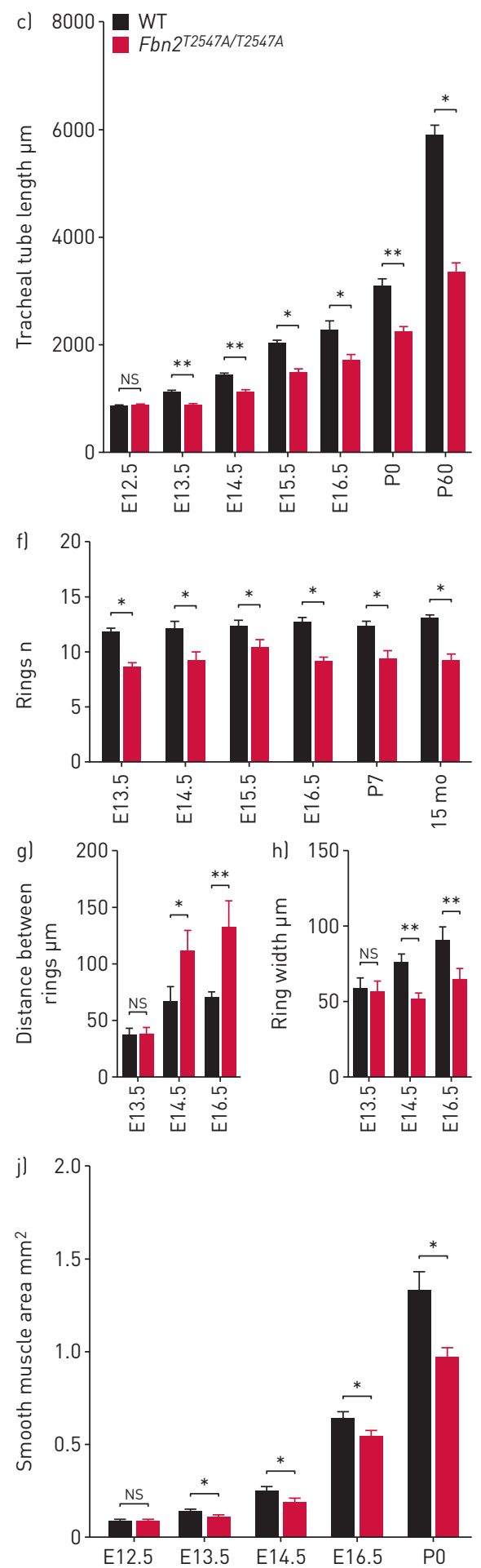

FIGURE 3 Fbn2 $2^{T 2547 A / T 2547 A}$ mice display defects in tracheal elongation, cartilage ring formation and smooth muscle organisation. al Representative images of ventral views of wild-type (WT) $(n=6)$ and Fbn2 ${ }^{T 2547 A / T 2547 A}(n=6)$ tracheas at several embryonic stages. Double-ended arrows indicate tracheal tube length. Scale bar $=1 \mathrm{~mm}$. b) Representative images of ventral views of WT (n=6) and Fbn2 ${ }^{T 2547 A / T 2547 A}(\mathrm{n}=6)$ tracheas at postnatal

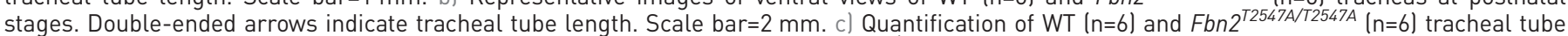
length. d) Ventral views of SOX9 immunostaining (green) of WT ( $\mathrm{n}=7$ ) and Fbn2 ${ }^{\text {T2547A/T2547A }}$ ( $\mathrm{n}=7$ ) tracheas at several embryonic stages. Arrows point to tracheal rings. Scale bar=1 mm. e) Representative images of ventral views of wholemount tracheas stained with alcian blue from 15-month-old

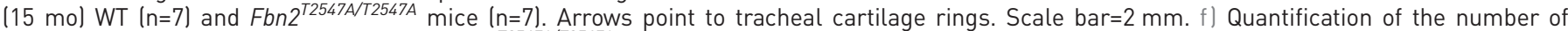

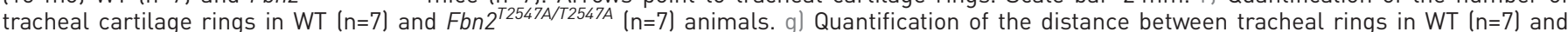
Fbn2 ${ }^{\text {T2547A/T2547A }}\left(\mathrm{n}=7\right.$ ) animals. h) Quantification of the ring width in WT ( $\mathrm{n}=7$ ) and Fbn2 ${ }^{\text {T2547A/T2547A }}$ (n=7) animals. i) Dorsal views of $\alpha$-smooth muscle actin (SMA) immunostaining (red) of WT $(n=6)$ and Fbn2 $2^{\text {T2547A/T2547A }}$ ( $n=6$ ) tracheas at several embryonic and postnatal stages. Scale bar $=100 \mu \mathrm{m}$. j) Quantification of smooth muscle area in WT (n=6) and Fbn2 $2^{T 2547 A / T 2547 A}$ (n=6) animals. E: embryonic day; P: postnatal day. Data are presented as mean \pm SD. ${ }^{*}: p<0.05 ;{ }^{* *}: p<0.01$; NS: nonsignificant; unpaired t-test. 
round shapes and progressively develop into spindle-shaped cells that circumferentially align the tube by E13.5 (figure $4 \mathrm{a}$ and $\mathrm{b}$ ). We observed no significant differences in smooth muscle alignment between Fbn2 $2^{T 2547 A / T 2547 A}$ and WT tracheas at E12.5 (figure 4c and d). However, at E13.5, Fbn2 $2^{\text {T2547A/T2547A }}$ smooth muscle cells displayed random alignment (figure $4 \mathrm{c}$ and $\mathrm{d}$ ), and this phenotype was observed in adult animals (figure $4 \mathrm{c}$ and $\mathrm{d}$ ). This smooth muscle phenotype temporally correlates with the onset of the tracheal elongation defects in Fbn2 $2^{T 2547 A / T 2547 A}$ mice (figure $3 \mathrm{C}$ and d). Correct orientation of the cell body has been shown to depend on the establishment of cell polarity [37]. To better understand the polarisation of smooth muscle cells, we examined the localisation of the Golgi apparatus relative to the cell nucleus, using the cis-Golgi matrix marker GM130, a widely used method to determine cell polarity in various cell types $[38,39]$. In WT smooth muscle cells, the GM130-labeled Golgi localises preferentially by the long edges of the nucleus (figure $4 \mathrm{e}$ and $\mathrm{f}$ ). In contrast, in Fbn2 $2^{T 2547 A / T 2547 A}$ smooth muscle cells, the Golgi exhibited random alignment (figure $4 \mathrm{e}$ and $\mathrm{f}$ ). Tracheal smooth muscle cells establish polarity before E12.5, and its disruption causes altered smooth muscle architecture by E12.5 [36]. Fbn2 $2^{T 2547 A / T 2547 A}$ smooth muscle exhibited no significant differences compared to WT at E12.5 (figure 4c and d). These results indicate that $\mathrm{Fbn} 2$ is dispensable for the initiation of smooth muscle cell polarity, but becomes necessary for its maintenance. Tissue contractility has been reported to drive tubulogenesis [3, 40]. Based on the findings that Fbn2 is required for smooth muscle cell alignment and polarity, we hypothesised that a disruption in smooth muscle cell orientation might lead to compromised circumferential tracheal contraction contributing to tube elongation defects in Fbn2 mutant mice. Interestingly, Fbn2 $2^{\text {T2547A/T2547A }}$ tracheas exhibited increased duration (figure $4 \mathrm{~g}$ and $\mathrm{h}$ and supplementary movie 1 ) and reduced amplitude (figure $4 \mathrm{~g}$ and $\mathrm{i}$ and supplementary movie 1) of spontaneous contractions compared to WT at E13.5. In addition, $\mathrm{Fbn2} 2^{T 2547 \mathrm{~A} / T 2547 \mathrm{~A}}$ tracheas exhibited significantly reduced contractile forces compared to WT (figure 4j). These findings indicate that FBN2 is essential to maintain smooth muscle cell polarity to direct tissue architecture and contractility, ultimately driving tracheal tube elongation.

The oesophagus and trachea separate from a common foregut tube at around E9.5 [41]. Thus, we sought to determine whether Fbn2 was also required for oesophageal tube formation. FBN2 is highly expressed in oesophageal smooth muscle (supplementary figure S13a), but we found no significant difference in tube length between $F b n 2^{T 2547 A / T 2547 A}$ and WT mice (supplementary figure S13b and c). Next, we examined oesophageal smooth muscle morphology. Smooth muscle cells are localised in the outer layers of the oesophageal wall [42] (supplementary figure S13a and d), and spindle-shaped smooth muscle cells are circumferentially aligned by E14.5 (supplementary figure S13e and f). Unlike in the trachea, we observed no obvious differences in oesophageal smooth muscle organisation (supplementary figure S13d) or smooth

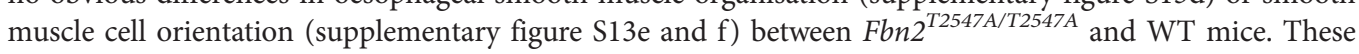
data indicate that $\mathrm{Fbn} 2$ is dispensable for smooth muscle development during oesophageal tube formation.

\section{Fbn2 modulates elastic fibre formation and Fibronectin accumulation}

To investigate the molecular mechanisms underlying Fbn2-mediated tracheal tube formation, we examined ECM protein formation and levels. Fibrillin microfibrils are known to provide a scaffold for the deposition of Tropoelastin, which matures to Elastin and eventually to elastic fibres [43-47]. We hypothesised that Fbn2 deficiency might lead to defects in Elastin structure. At E14.5, elastic fibres around WT smooth muscle cells were aligned parallel to the long edges of the nuclei and approximately perpendicular to the direction of tube elongation (figure $5 \mathrm{a}$ and b). In contrast, elastic fibre orientation in Fbn2 $2^{\text {T2547A/T2547A }}$ tracheas appeared random, with most of them oriented at an angle oblique to the direction of tube elongation (figure 5a and b). Interestingly, short elastic fibres were also observed in Fbn2 $2^{\text {T2547A/T2547A }}$ tracheas, unlike in WT (figure 5a and c). However, Fbn2 $2^{T 2547 A / T 2547 A}$ tracheas exhibited no obvious changes in Elastin protein levels (figure 5d), indicating that FBN2 is required for elastic fibre formation but not Elastin protein expression. Next, we examined elastic fibres in oesophageal smooth muscle cells. There was no significant difference in elastic fibre formation between Fbn2 $2^{\text {T2547A/T2547A }}$ and WT oesophagi (supplementary figure S13g-i). Fbn1 has been reported to modulate elastic fibre formation [48-50]. However, Fbn2 null mutants exhibit WT-like vascular smooth muscle cells and elastic fibre formation [44]. Interestingly, Fbn1 is highly expressed in oesophagi (supplementary figure S13j) and FBN1 localises to blood vessel walls [51]. These results suggest that Fbn1 and Fbn2 may play redundant functions in oesophageal and vascular smooth muscle morphogenesis.

Another critical ECM protein, Fibronectin (FN), has been shown to bind FBN2 [52, 53] and promote cell elongation and alignment $[54,55]$. Interestingly, Fbn2 ${ }^{T 2547 A / T 2547 A}$ tracheas exhibited significantly decreased FN protein levels compared to WT (figure $5 \mathrm{~d}-\mathrm{g}$ ), indicating that FN accumulation is dependent on FBN2. However, we observed no significant difference in Fn mRNA levels in Fbn2 $2^{\text {T2547A/T2547A }}$ tracheas compared to WT (figure 5h). Methylamine hydrochloride, a lysosomal inhibitor, has been reported to inhibit FN degradation [56]. After treatment with methylamine hydrochloride, Fbn2 $2^{\text {T2547A/T2547A }}$ tracheas exhibited partially restored FN protein levels (figure $5 \mathrm{i}$ and $\mathrm{j}$ ), indicating that FBN2 protects FN from 

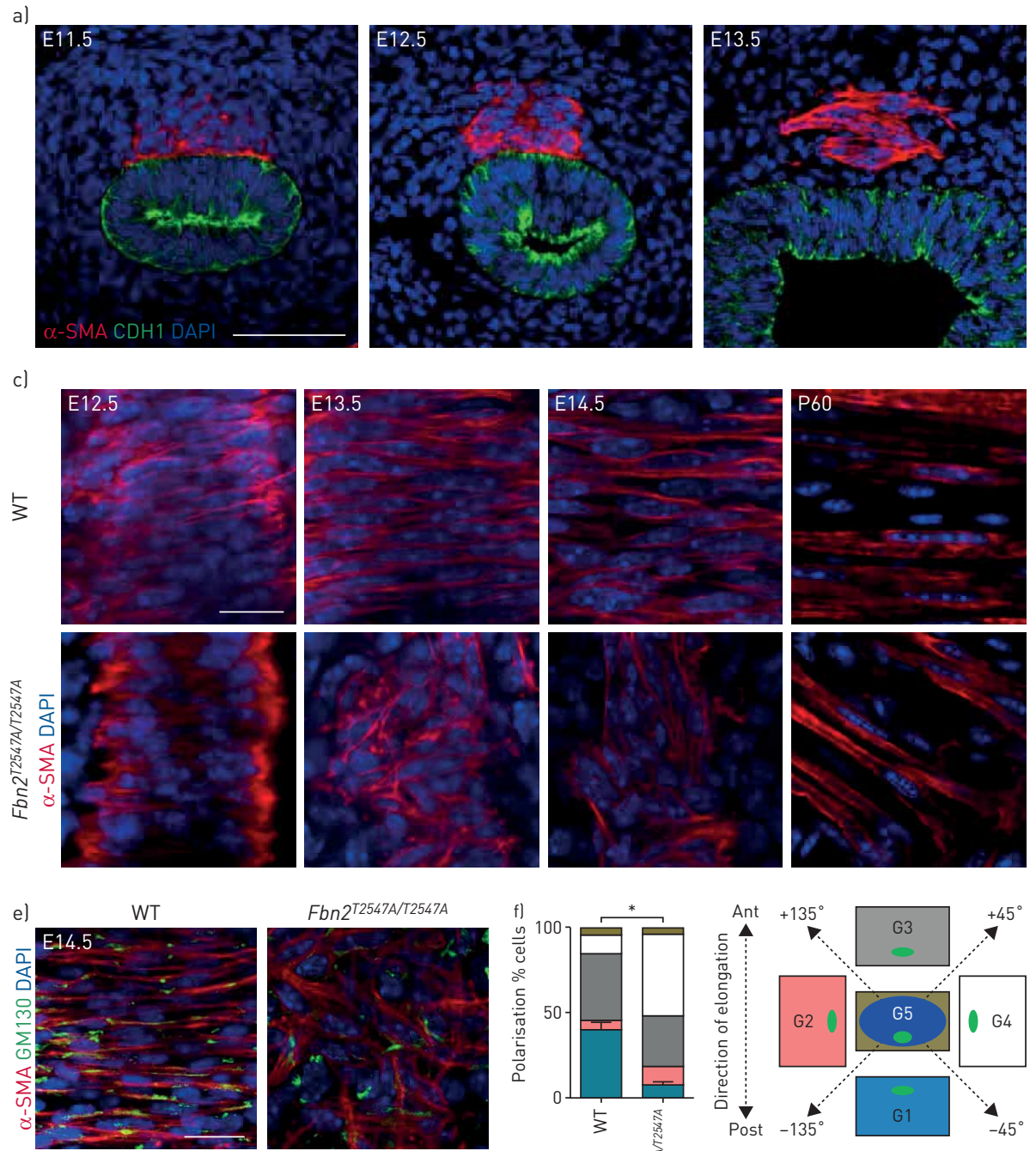

Fbn2T2547A/T2547A
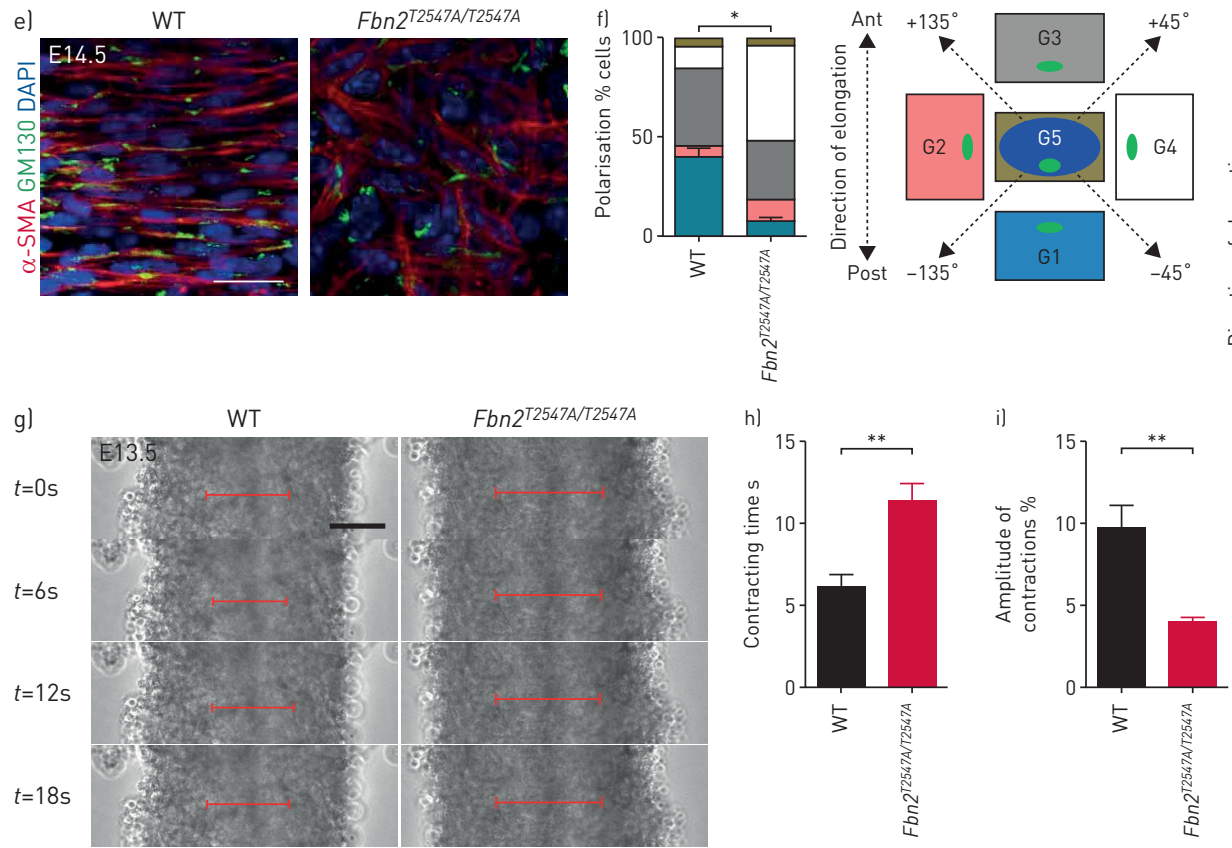
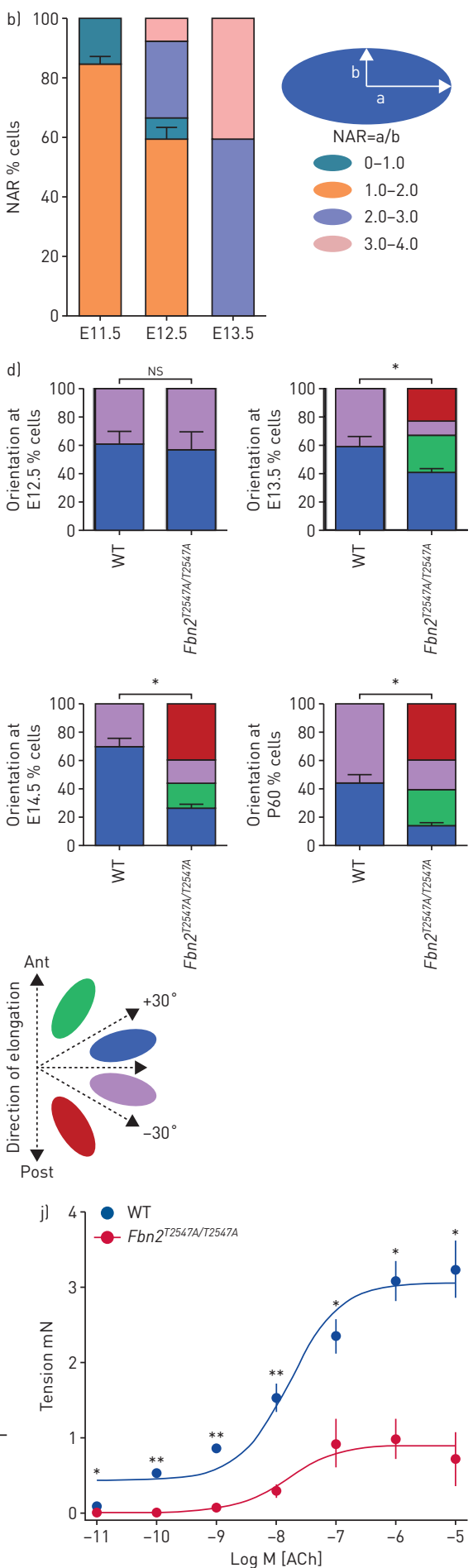

FIGURE 4 Fbn2 regulates smooth muscle cell orientation and polarisation. a) Immunostaining for $\alpha$-smooth muscle actin (SMA) (red) and CDH1 (green), and 4',6-diamidino-2-phenylindole (DAPI) staining (blue) of transverse sections of wild-type (WT) tracheas (n=6) at several embryonic stages. Scale bar $=50 \mu \mathrm{m}$. b) Quantification of tracheal smooth muscle cell nuclear aspect ratio (NAR). c) Dorsal views of $\alpha$-SMA immunostaining (red) and DAPI staining (blue) of WT $(n=7)$ and Fbn2 $2^{T 2547 A / T 2547 A}(n=7)$ tracheas at several embryonic $(E)$ and postnatal $(P)$ stages. Scale bar=20 $\mu$ m. d) Quantification of WT $(n=7)$ and Fbn2 ${ }^{T 2547 A / T 2547 A}(n=7)$ tracheal smooth muscle cell orientation. e) Dorsal views of $\alpha$-SMA (red) and GM130 (green) immunostaining and DAPI staining (blue) of E14.5 WT $(n=6)$ and Fbn2 ${ }^{T 2547 A / T 2547 A}(n=6)$ tracheas. Scale bar=20 $\mu m$. $f$ ) Quantification of E14.5

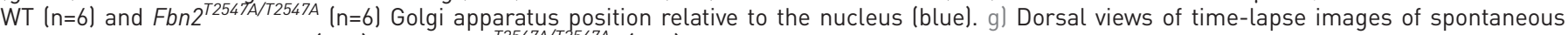
contractions of E13.5 WT $(n=6)$ and Fbn2 $22547 \mathrm{~A} / \mathrm{T2547 \textrm {A }}(\mathrm{n}=6)$ tracheas. Lines indicate tracheal smooth muscle width. Scale bar=50 $\mu \mathrm{m}$. h) Quantification of contracting time of E13.5 WT $(n=6)$ and Fbn2 $2^{T 2547 A / T 2547 A}(n=6)$ tracheas. i) Quantification of the amplitude of contractions of

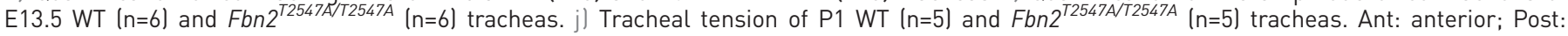
posterior. Data are presented as mean \pm SD. ${ }^{*}: p<0.05 ;{ }^{* *}$ : $p<0.01$; NS: nonsignificant; unpaired t-test. 

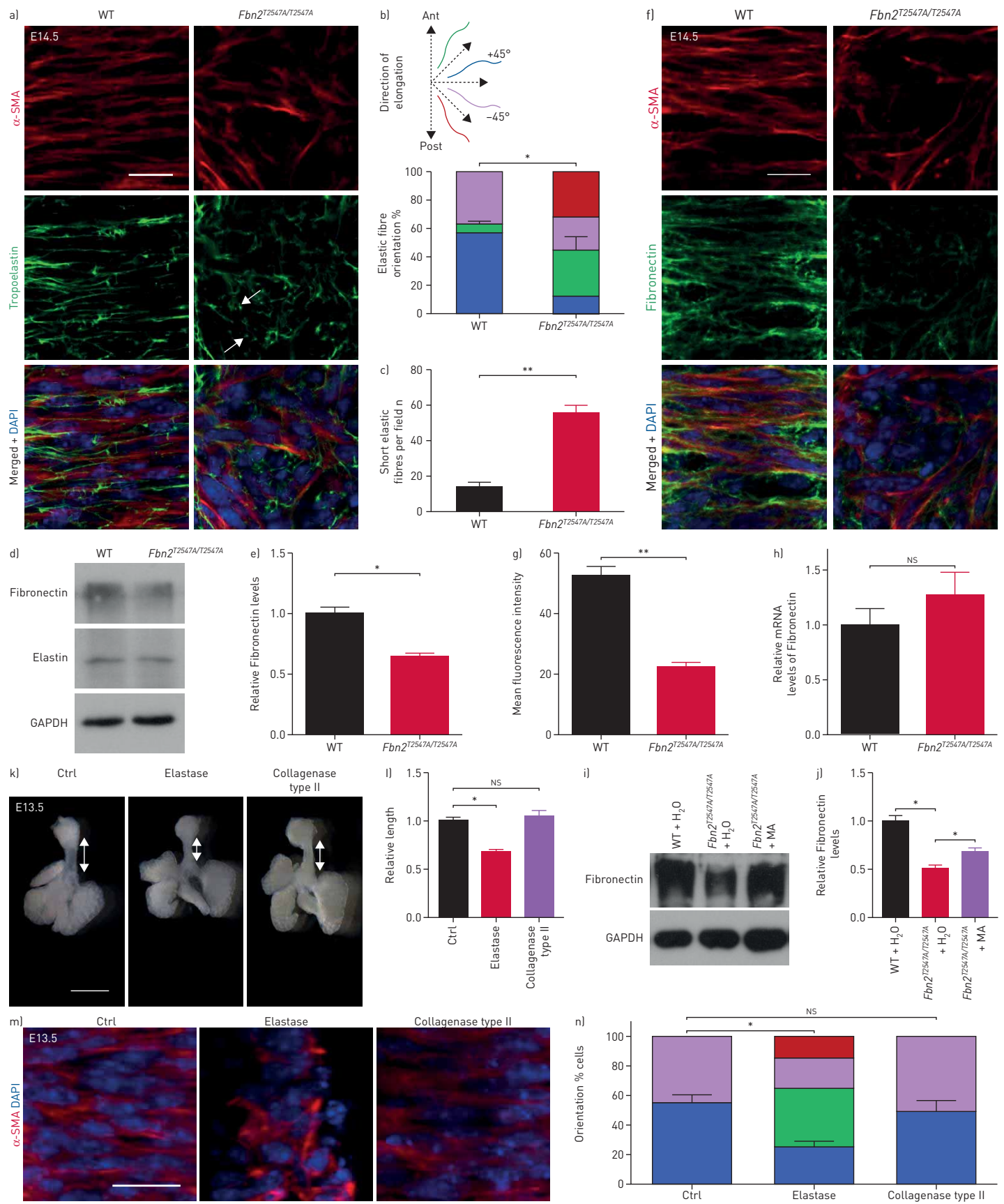

FIGURE 5 Fbn2 modulates elastic fibre formation and Fibronectin accumulation. a) Dorsal views of $\alpha$-smooth muscle actin (SMA) (red) and Tropoelastin (green) immunostaining and 4',6-diamidino-2-phenylindole (DAPI) staining (blue) of embryonic day (E)14.5 wild-type (WT) ( $\mathrm{n}=8$ ) and Fbn2 ${ }^{\text {T2547A/T2547A }}(\mathrm{n}=8)$ tracheas. Arrows point to short elastic fibres. Scale bar $=20 \mu \mathrm{m}$. Quantification of b) elastic fibre orientation and c) short

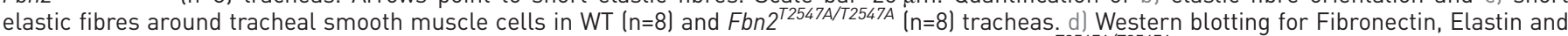
glyceraldehyde 3-phosphate dehydrogenase (GAPDH) in postnatal day (P)7 WT (n=7) and Fbn2 $2^{\text {T2547A/T2547A }}$ ( $\mathrm{n}=7$ ) tracheas. e) Quantification of relative levels of Fibronectin in P7 WT $(n=7)$ and Fbn2 $2547 A / T 2547 A$ ( $n=7)$ tracheas. f) Dorsal views of $\alpha$-SMA (red) and Fibronectin (green) immunostaining and DAPI staining (blue) of E14.5 WT $(n=8)$ and Fbn2 $2547 \mathrm{~A} / T 2547 \mathrm{~A}$ ( $\mathrm{n}=8$ ) tracheas. Scale bar=20 $\mu \mathrm{m}$. g) Quantification of mean

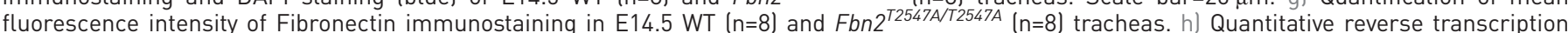

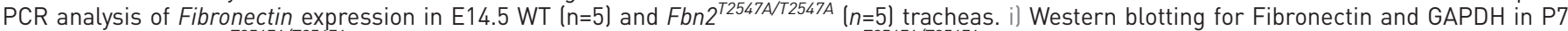
WT $(n=5)$ and Fbn2 $2^{\text {T2547A/T2547A }}(n=5) \quad d_{d H_{2}} 0$-treated tracheas, and in Fbn2 T2547A/T2547A methylamine hydrochloride-treated tracheas (n $\left.=5\right)$.

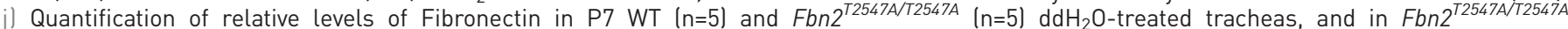
methylamine hydrochloride (MA)-treated tracheas $(n=5) . k$ ) Representative images of ventral views of E13.5 tracheas after treatment for $28 \mathrm{~h}$ with DMEM/F-12 medium ( $\mathrm{n}=6), 2 \mathrm{mU} \cdot \mathrm{mL}^{-1}$ elastase $(\mathrm{n}=6)$ or $2 \mathrm{mU} \cdot \mathrm{mL}^{-1}$ collagenase type II $(\mathrm{n}=6)$. Scale bar=1 mm. () Quantification of relative tracheal tube length after treatment for $28 \mathrm{~h}$ with DMEM/F-12 medium ( $\mathrm{n}=6), 2 \mathrm{mU} \cdot \mathrm{mL}^{-1}$ elastase ( $\mathrm{n}=6$ ) or $2 \mathrm{mU} \cdot \mathrm{mL}^{-1} \mathrm{collagenase}$ type II ( $\mathrm{n}=6$ ). m) Dorsal views of $\alpha$-SMA immunostaining (red) and DAPI staining (blue) of E13.5 tracheas after treatment for $28 \mathrm{~h}$ with DMEM/F-12 medium $(\mathrm{n}=6), 2 \mathrm{mU} \cdot \mathrm{mL}^{-1}$ elastase $(\mathrm{n}=6)$ or $2 \mathrm{mU} \cdot \mathrm{mL}^{-1}$ collagenase type II (n=6). Scale bar $=20 \mu \mathrm{m}$. n) Quantification of tracheal smooth muscle cell orientation after treatment for $28 \mathrm{~h}$ with DMEM/F-12 medium $(\mathrm{n}=6)$ or $2 \mathrm{mU} \cdot \mathrm{mL}^{-1}$ elastase $(\mathrm{n}=6)$ or $2 \mathrm{mU} \cdot \mathrm{mL}^{-1} \mathrm{collagenase}$ type $\|$ ( $\mathrm{n}=6$ ). Ant: anterior; Post: posterior. Data are presented as mean \pm SD. ${ }^{*}: p<0.05 ;{ }^{* *}$ : $p<0.01$; NS: nonsignificant; unpaired t-test. 
lysosomal degradation during tracheal tube formation. To test whether FBN2 plays a role in elastic fibre formation and FN accumulation in adult mice, we examined elastic fibres and FN in adult tracheas. We found that $F b n 2^{T 2547 A / T 2547 A}$ P70 tracheas exhibited random elastic fibre orientation (supplementary figure S14a and b), short elastic fibres (supplementary figure S14a and c), decreased FN protein levels (supplementary figure S14d and e), but no obvious difference in Elastin protein levels (supplementary figure S14f and g) compared to WT, possibly suggesting that FBN2 is needed for ECM homeostasis in mature mice. However, to examine the adult function of Fbn2, it will be necessary to generate and use a floxed Fbn2 allele. Elastin deficiency has been reported to alter the orientation of smooth muscle cells in the vessel wall $[57,58]$. We thus hypothesised that disruption of elastic fibres might phenocopy the Fbn2 deficiency-induced tracheal elongation and smooth muscle cell orientation defects. Interestingly, after treatment for $28 \mathrm{~h}$ with $2 \mathrm{mU} \cdot \mathrm{mL}^{-1}$ elastase, E13.5 tracheas exhibited a significant reduction in tube length (figure 5k and 1 ) and altered smooth muscle cell orientation (figure $5 \mathrm{~m}$ and $\mathrm{n}$ ) compared to controls, phenotypes that were not observed after treatment with collagenase type II (figure $5 \mathrm{k}-\mathrm{n}$ ). Altogether, these results suggest that FBN2-mediated ECM formation and accumulation are essential for tracheal smooth muscle cell orientation and organ elongation.

\section{p38-dependent downregulation of Mmp expression as a mediator of Fbn2 function in tracheal development}

We sought to further understand how FBN2 deficiency leads to impaired ECM formation and accumulation. The observation of short elastic fibres and FN degradation prompted us to analyse the levels of MMP2 and MMP9, key Elastin and Fibronectin-cleaving enzymes [59-62]. Fbn2 $2^{\text {T2547A/T2547A }}$ tracheas exhibited significantly increased $M m p 2$ and $M m p 9$ mRNA levels (figure 6a and supplementary table S1) as well as increased protein levels (figure $6 \mathrm{~b}$ and c), and activity (figure $6 \mathrm{~d}$ and e). In contrast, $\mathrm{Fbn2} 2^{\text {T2547A/T2547A }}$ oesophagi exhibited no obvious difference in Mmp2 mRNA levels (supplementary figure S15a and supplementary table S1), while Mmp9 mRNA was undetectable in E14.5 WT oesophagi (supplementary table S1). MMP inhibition by GM6001 [63] has been shown to reduce Elastin cleavage [64] and FN degradation [65]. We thus tested whether MMP inhibition could rescue the Fbn2 $2^{\text {T2547A/T2547A }}$ tracheal phenotypes. We administered GM6001 $\left(50 \mathrm{mg} \cdot \mathrm{kg}^{-1}\right)$ intraperitoneally for three consecutive days from E12.5 to E14.5. Interestingly, after GM6001 injection, Fbn2 $2^{T 2547 A / T 2547 A}$ tracheas exhibited partially rescued tube elongation (figure $6 \mathrm{f}$ and $\mathrm{g}$ ) and smooth muscle orientation (figure $6 \mathrm{~h}$ and i) phenotypes, as well as partially restored elastic fibre formation (figure $6 \mathrm{~h}, \mathrm{j}$ and $\mathrm{k}$ ) and $\mathrm{FN}$ accumulation (figure $6 \mathrm{~h}$ and $\mathrm{l}$ ) as assessed in a blind analysis. We aimed to find mechanisms by which $F b n 2$ regulates $M m p 2$ and $M m p 9$ expression and thus examined the expression of Atf2 and Hspb1, potential regulators of Mmp2 expression [66], as well as Nfkb1, Nfkb2, Rela, Jun and Fos, potential regulators of Mmp9 expression [66]. Fbn2 $2^{\text {T2547A/T2547A }}$ tracheas exhibited WT-like expression levels of these genes (supplementary figure S15b,c and supplementary table S1), indicating that Fbn2 modulates Mmp2 and Mmp9 mRNA levels through other pathways.

Mmps have been identified to be downstream targets of p38 MAPK signalling [67], and inhibition of the p38 MAPK signalling pathway efficiently downregulates $M m p 2$ and $M m p 9$ expression [67, 68]. Thus, we hypothesised that Fbn2 deficiency might lead to increased p38 MAPK activity. We observed that levels of phosphorylated p38 were greatly elevated in Fbn2 $2^{T 2547 A / T 2547 A}$ tracheas compared to WT (figure 7a and b and supplementary figure S16a and b), a phenotype that was not observed in Fbn2 $2^{\text {T2547A/T2547A }}$ oesophagi (supplementary figure S16c and d). In addition, after treatment with SB203580, a small molecule that decreases p38 phosphorylation [69], $F b n 2^{T 2547 A / T 2547 A}$ tracheas exhibited partially restored $M m p 2$ and Mmp9 mRNA levels (figure 7c and supplementary table S1). We then administered SB203580 $\left(1 \mu \mathrm{M}^{\mathrm{kg}} \mathrm{kg}^{-1}\right)$ intraperitoneally for three consecutive days from E12.5 to E14.5. Notably, after SB203580 injection, Fbn2 ${ }^{\text {T2547A/T2547A }}$ tracheas exhibited partially rescued tube elongation (figure $7 \mathrm{~d}$ and e) and smooth muscle orientation (figure $7 \mathrm{f}$ and $\mathrm{g}$ ) phenotypes, restored elastic fibre formation (figure $7 \mathrm{f}, \mathrm{h}$ and $\mathrm{i}$ ) as well as $\mathrm{FN}$ accumulation (figure $7 \mathrm{f}$ and $\mathrm{j}$ ) as assessed in a blind analysis. These results indicate that an increase in p38 phosphorylation-mediated $M m p$ levels in Fbn2 $2^{\text {T2547A/T2547A }}$ tracheas partially accounts for the smooth muscle cell orientation phenotype via its action on ECM homeostasis (figure $7 \mathrm{k}$ ).

\section{FBN2 and Fibronectin are decreased in the tracheas of human tracheomalacia patients}

Since Fbn $2^{T 2547 A / T 2547 A}$ mice exhibited tracheal cartilage defects, phenotypes similar to those observed in tracheomalacia patients (figure 8a), we examined FBN2 expression in tracheas from healthy controls and tracheomalacia patients (supplementary table 2). FBN2 localised to the peripheral regions of tracheal cartilage (figure $8 \mathrm{~b}$ ) and did not surround $\mathrm{SOX}^{+}$mesenchymal cells (figure $8 \mathrm{~b}$ ) in postnatal human tracheas, expression patterns similar to those observed in mice at postnatal stages (figure $2 \mathrm{~b}$ ). We observed that FBN2 levels were significantly decreased in the cartilage regions of tracheas from tracheomalacia patients compared to healthy controls (figure $8 \mathrm{~b}$ and c). We also examined Fibronectin expression. Fibronectin localised to the peripheral regions of tracheal cartilage (figure $8 \mathrm{~d}$ ) and did not surround 

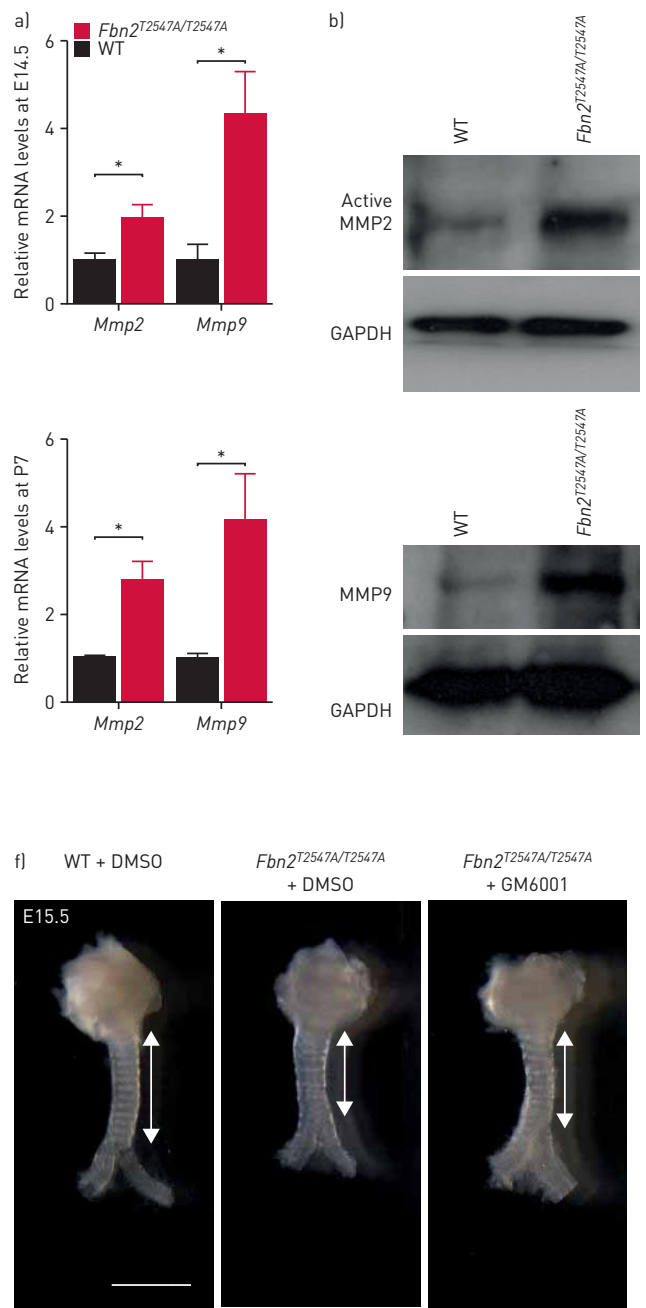

h)

WT + DMSO

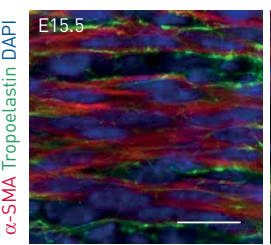

Fbn2 $2^{\text {T2547A/T254 }}$

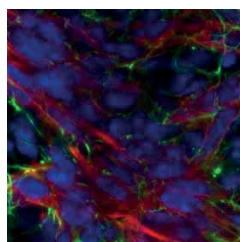

Fbn2T2547ATT2547A
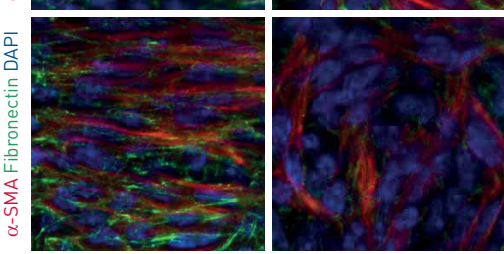

+ GM6001
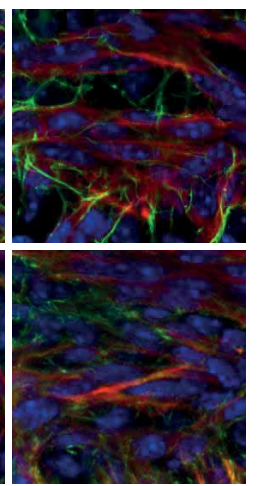
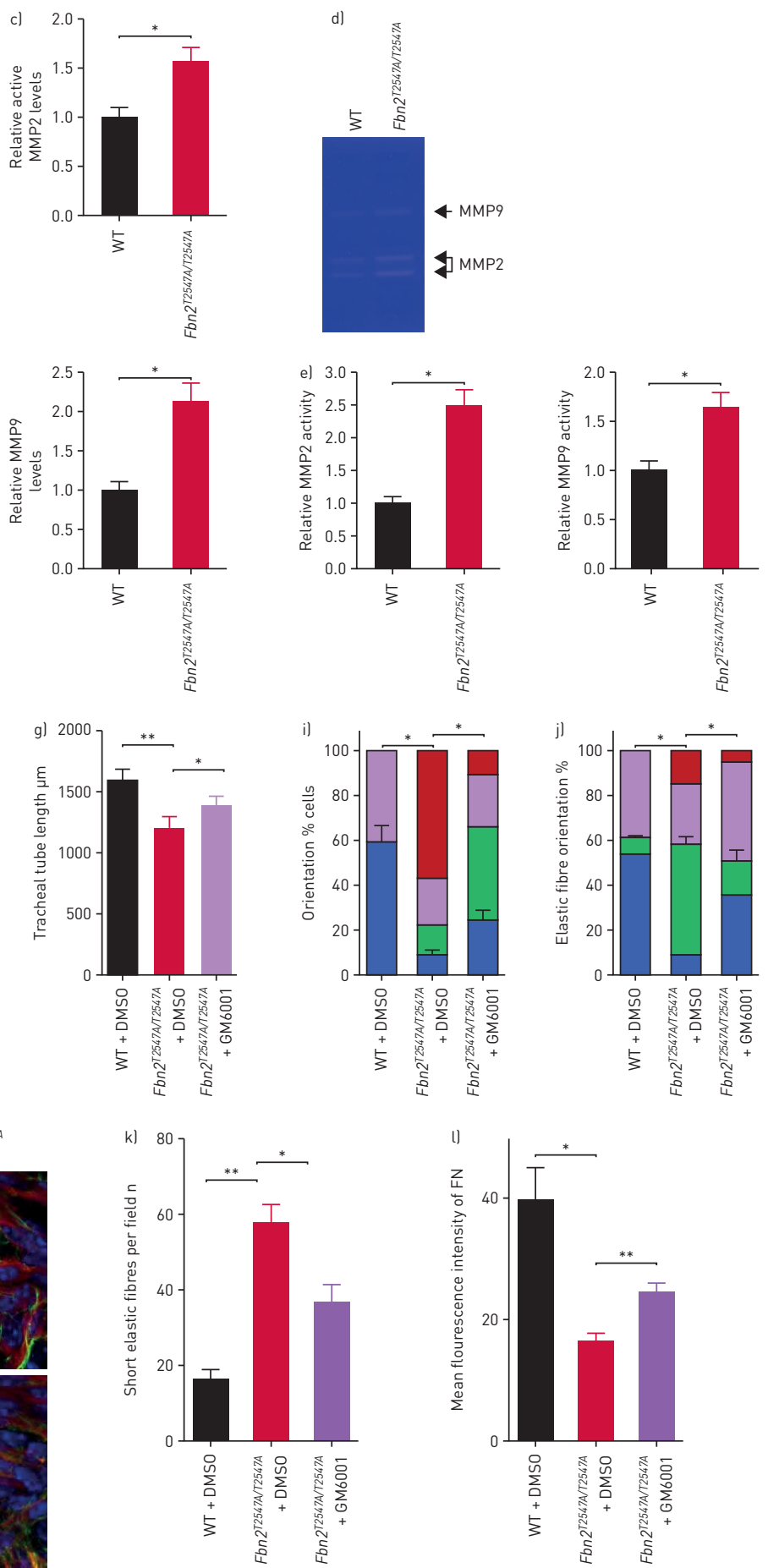

FIGURE 6 Matrix metalloproteinases (MMPs) function as mediators of Fibrillin (FBN)2 function in tracheal development. a) Quantitative reverse transcription PCR analysis of Mmp2 and Mmp9 expression in embryonic day (E)14.5 and postnatal day (P)7 wild-type (WT) (n=5 and 5) and

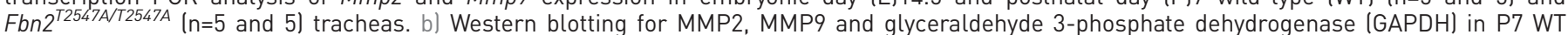

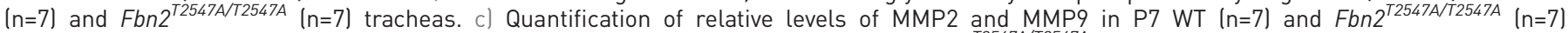

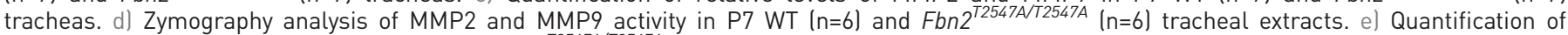

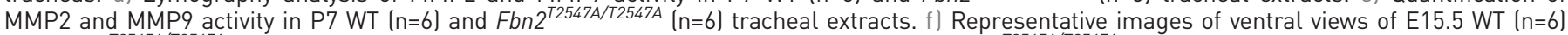

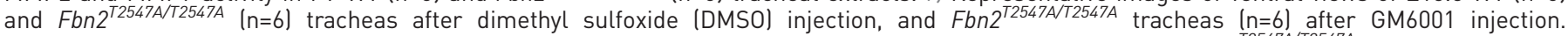

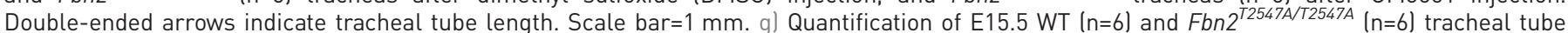
length after DMSO injection, and Fbn2 $2^{\text {T2547A/T2547A }}$ tracheas (n=6) after GM6001 injection. h) Dorsal views of $\alpha$-smooth muscle actin (SMA) (red) and Tropoelastin (green), or Fibronectin (green) immunostaining and 4',6-diamidino-2-phenylindole (DAPI) staining (blue) of E15.5 WT (n=6) and

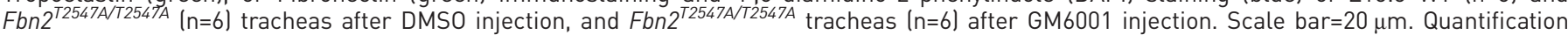
of i) tracheal smooth muscle cell orientation, j) elastic fibre orientation, kJ short elastic fibres and (] mean fluorescence intensity of Fibronectin immunostaining in E15.5 WT $(n=6)$ and Fbn2 ${ }^{T 2547 A / T 2547 A}(\mathrm{n}=6)$ tracheas after DMSO injection, and in Fbn2 ${ }^{T 2547 A / T 2547 A}$ tracheas (n=6) after GM6001 injection. Data are presented as mean \pm SD. ${ }^{*}: p<0.05 ;{ }^{* *}: p<0.01$; unpaired t-test. 

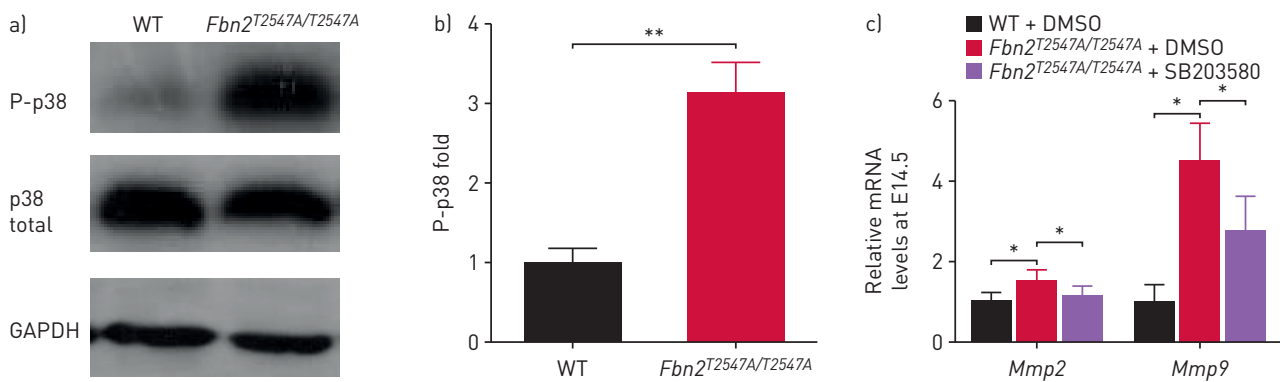

d) $\mathrm{WT}+\mathrm{DMSO}$

Fbn2T2547A/T2547A + DMSO
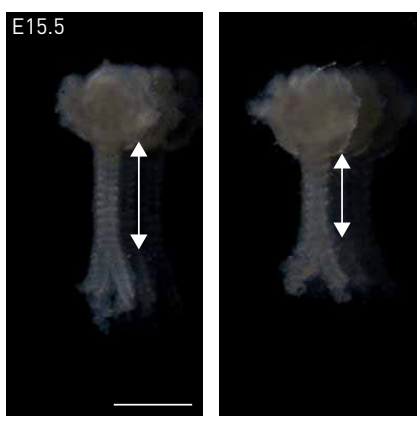

Fbn2T2547A/T2547A + SB203580

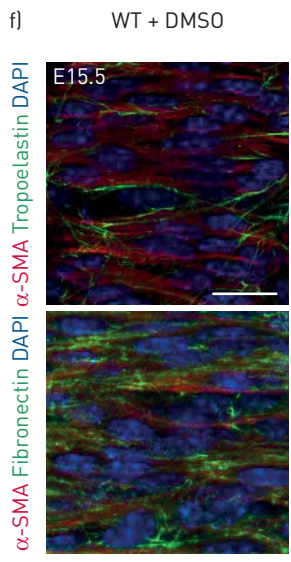

Fbn2T2547A/T2547A

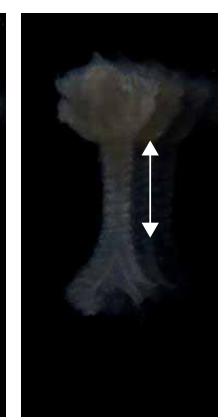

e) 2000

Mmp2

Mmp9

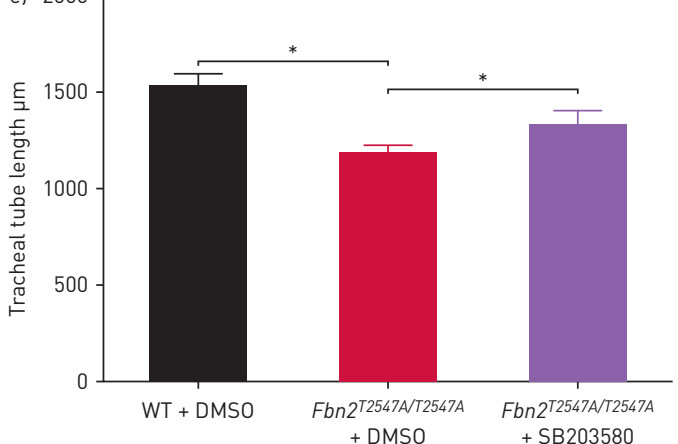

k)

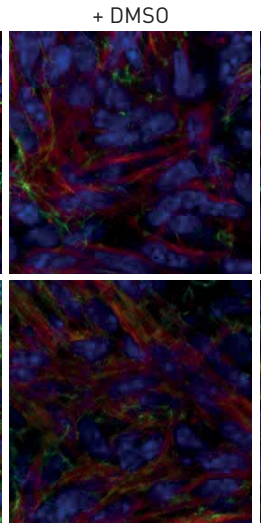

$b n 2^{T 2547 A / T 2547 A}$

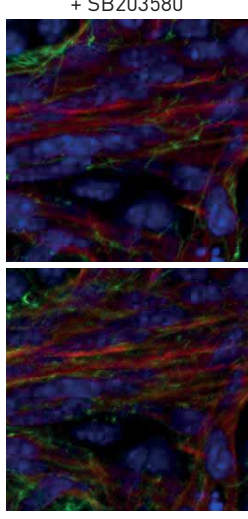

Fbn2T2547A/T2547A

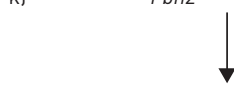

Reduced FBN2 deposition

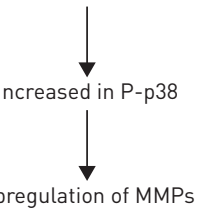

Defects in ECM formation and deposition
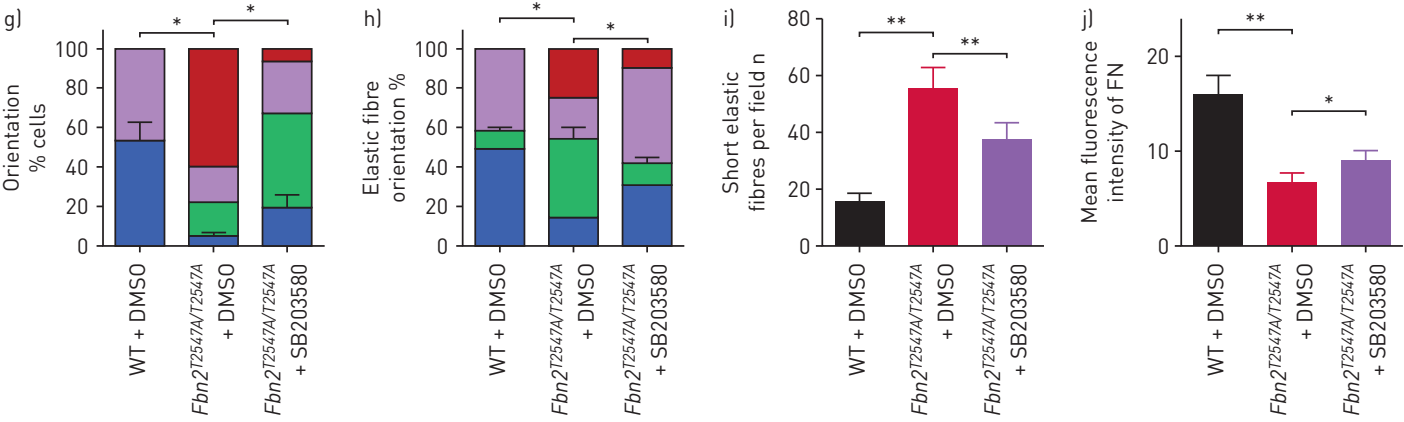

FIGURE 7 Increased p38 signalling correlates with increased Matrix metalloproteinases (Mmp) expression, impaired elastic fibre formation and Fibronectin degradation. a) Western blotting for phospho-p38, total p38 and glyceraldehyde 3-phosphate dehydrogenase (GAPDH) in postnatal day

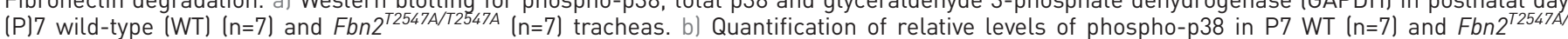
T2547A (n=7) tracheas. c) Quantitative reverse transcription PCR analysis of Mmp2 and Mmp9 expression in embryonic day (E)14.5 WT (n=5) and Fbn2 $2^{\text {T2547A/T2547A }(n=5) ~ D M S O-t r e a t e d ~ t r a c h e a s, ~ a n d ~ i n ~ F b n 2 ~} 2$ T257A/T2547A SB203580-treated tracheas ( $\left.n=5\right)$. d) Representative images of ventral views

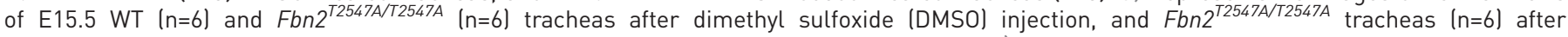
SB203580 injection. Double-ended arrows indicate tracheal tube length. Scale bar=1 mm. el Quantification of tracheal tube length in E15.5 WT $(\mathrm{n}=6)$ and Fbn2 $2^{\text {T2547A T2547A }}(\mathrm{n}=6)$ after DMSO injection, and in Fbn2 $2^{\text {T2547A/T2547A }}(\mathrm{n}=6)$ after SB203580 injection. $\mathrm{f}$ ) Dorsal views of $\alpha$-smooth muscle actin (SMA) (red) and Tropoelastin (green), or Fibronectin (green) immunostaining and $4^{\prime}, 6$-diamidino-2-phenylindole (DAPI) staining (blue) of E15.5 WT $(n=6)$ and Fbn2 ${ }^{\text {T2547A/T2547A }(n=6) \text { tracheas after DMSO injection, and Fbn2 } 2547 A / T 2547 A}$ tracheas $(n=6)$ after SB203580 injection. Scale bar $=20 \mu \mathrm{m}$. Quantification of $\mathrm{g}$ ) tracheal smooth muscle cell orientation, h) elastic fibre orientation, i) short elastic fibres and j] mean fluorescence intensity of Fibronectin immunostaining in E15.5 WT $(n=6)$ and Fbn2 $2^{\text {T2547A/T2547A }}(n=6)$ tracheas after DMSO injection, and in Fbn2 $2^{\text {T2547A/T2547A }}$ tracheas $(n=6)$ after SB203580 injection. k) Proposed model for the effects of the Fbn2 $2^{T 2547 A}$ mutation on extracellular matrix (ECM) organisation and stability, and tracheal smooth muscle cell orientation. Data are presented as mean \pm SD. ${ }^{*}$ : $p<0.05 ;{ }^{* *}$ : $p<0.01$; unpaired t-test. 
a)

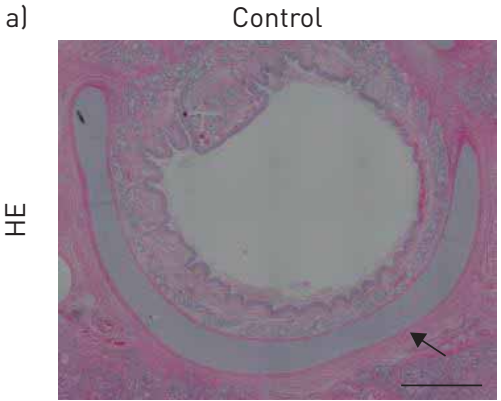

b)
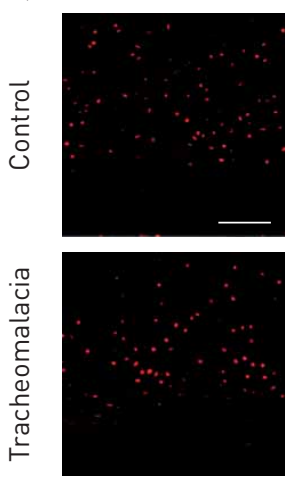

d)
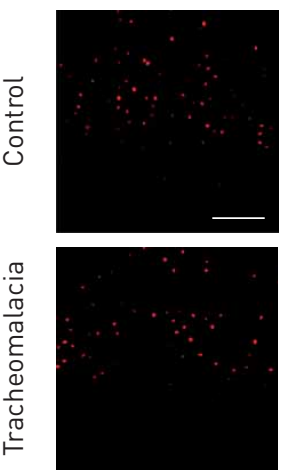

FBN2
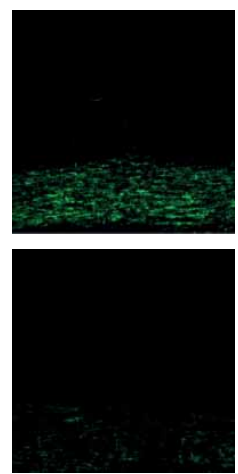

Fibronectin
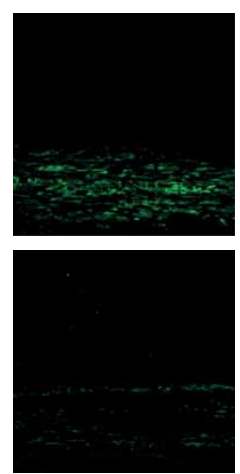
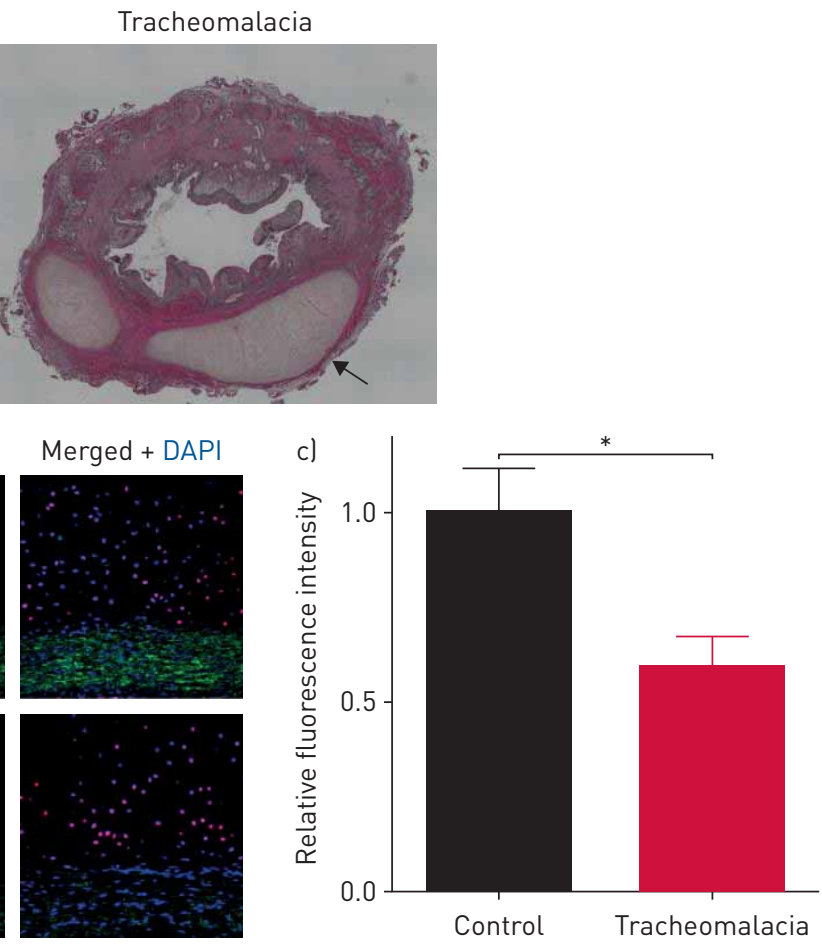

Merged + DAPI
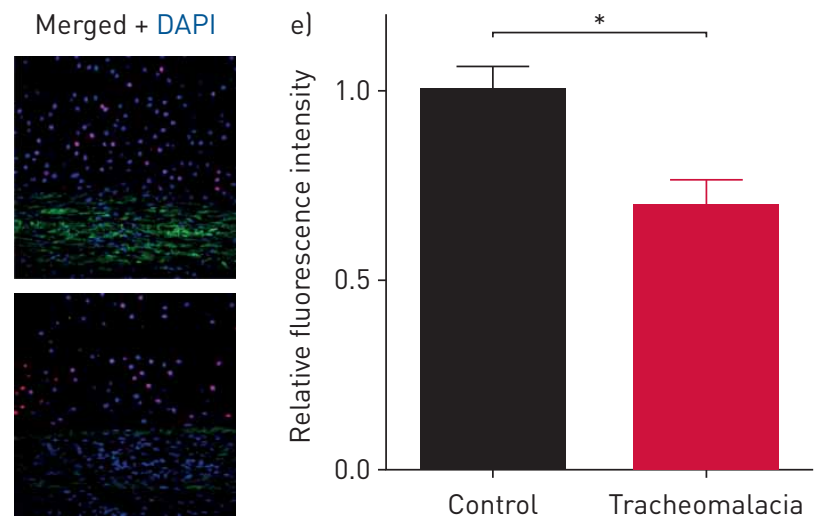

FIGURE 8 FBN2 and Fibronectin levels are decreased in the tracheas of human tracheomalacia patients. a) Representative images of transverse sections of tracheas stained with haematoxylin and eosin from healthy controls $(n=5)$ and tracheomalacia patients $(n=7)$. Arrows point to tracheal cartilage. Scale bar=1 mm. b) Immunostaining for SOX9 (red) and FBN2 (green), and 4',6-diamidino-2-phenylindole (DAPI) staining (blue) of transverse sections of ventral parts of tracheas from healthy controls $(n=4)$ and tracheomalacia patients $(n=6)$. Scale bar $=100 \mu \mathrm{m}$. c) Quantification of relative mean fluorescence intensity of FBN2 immunostaining in ventral parts of tracheas from healthy controls $(n=4)$ and tracheomalacia patients $(n=6)$. d) Immunostaining for SOX9 (red) and Fibronectin (green), and DAPI staining (blue) of transverse sections of ventral parts of tracheas from healthy controls $(n=5)$ and tracheomalacia patients $(n=5)$. Scale bar $=100 \mu \mathrm{m}$. e) Quantification of relative mean fluorescence intensity of Fibronectin immunostaining in ventral parts of tracheas from healthy controls $(n=5)$ and tracheomalacia patients $(n=5)$. Data are presented as mean $\pm S D .^{*}$ : $p<0.05$; unpaired t-test.

$\mathrm{SOX}^{+}$mesenchymal cells (figure 8d) in postnatal human tracheas, expression patterns similar to those observed in mice at postnatal stages (supplementary figure S17). Interestingly, Fibronectin levels also appeared to be reduced in the cartilage regions of tracheas from tracheomalacia patients compared to healthy controls (figure $8 \mathrm{~d}$ and e). These results in humans are consistent with findings in mice, suggesting that a decrease in ECM proteins, including FBN2 and Fibronectin, is associated with, and may contribute to, symptoms of tracheomalacia.

\section{Discussion}

Understanding tracheal tubulogenesis is a fundamental goal in the field of pulmonary development and disease, as conditions such as primary tracheomalacia and congenital tracheal stenosis arise due to tracheal 
tube formation defects. Our work suggests critical roles for smooth muscle cells and chondrocytes in tracheal tube formation. Differentiated smooth muscle cells elongate in a direction perpendicular to the axis of tracheal elongation and generate circumferential contractile force which promotes tube elongation. In Fbn2 $2^{T 2547 A / T 2547 A}$ tracheas, aberrantly oriented smooth muscle cells restrict tube elongation by compressing the mesenchyme and attached epithelium, as in the case of smooth muscle restriction of gut elongation [4]. Chondrocytes have also been reported to be essential for tracheal tube expansion [36, 70]. Notably, Sox 9 knockout mice exhibit defects in chondrocyte differentiation, tracheal cartilage formation and tracheal tube expansion [36, 70]. It is thus possible that in Fbn2 $2^{\text {T2547A/T2547A }}$ tracheas the chondrocyte differentiation defects lead to impaired cartilage formation, ultimately resulting in the narrowed tracheal

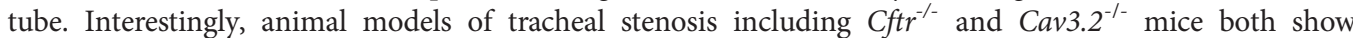
cartilage ring and lumen formation defects [71-73], phenotypes similar to those observed in Fbn2 $2^{\text {T2547A/ }}$ ${ }^{T 2547 A}$ mice. Cav3.2 regulates tracheal cartilage formation by promoting chondrocyte differentiation [73]. It is thus possible that a defect in chondrocyte differentiation is a common cause of congenital tracheal stenosis.

Here we reveal novel functions for FBN2 in elastic fibre formation and Fibronectin accumulation. Fbn2 mutants exhibit no significant differences in elastic fibre formation in oesophageal or vascular smooth muscle cells [44]. However, $\mathrm{Fbn1}^{-/-}$mice exhibit elastic fibre organisation defects in aortic tissues [44]. Fbn2 $2^{\text {T2547A/T2547A }}$ oesophagi exhibit WT-like p38 phosphorylation and Mmp2 mRNA expression levels. Fbn1 mutants exhibit increased p38 phosphorylation [74] as well as increased MMP2 and MMP9 levels in their thoracic aortas [75]. Interestingly, both $\mathrm{Fbn1}^{-/+} ; \mathrm{Fbn2}^{-/-}$and $\mathrm{Fbn1^{-1 }}$; and $\mathrm{Fbn2}^{-/-}$animals display a more severe vascular phenotype than $F b n 1^{-/-}$mice [44], indicating that $F b n 1$ and $F b n 2$ perform partially overlapping functions during aortic development. It is thus possible that the absence of ECM defects in Fbn2 $2^{T 2547 A / T 2547 A}$ oesophagi is due to functional redundancy with Fbn1. FBN1 promotes Tropoelastin coacervation [48], and prevents Elastin disruption and degradation possibly by decreasing MMP2 and MMP9 activity $[49,50]$. However, Fbn2 $2^{T 2547 A / T 2547 A}$ tracheas appear to exhibit no obvious reduction in Elastin protein levels, indicating that FBN2 is required for elastic fibre formation but not protein expression during tracheal tube formation. Fibrillin microfibrils have been reported to colocalise and interact with Fibronectin [52, 53], and their deposition depends on Fibronectin assembly [76]. Interestingly, our studies suggest that Fibrillin microfibrils are also required for Fibronectin accumulation.

Our study presents a novel signalling axis governing ECM homeostasis, through Fibrillin microfibrils, p38 mitogen-activated protein kinase (MAPK) activation and MMPs. Fbn2 deficiency leads to aberrant activation of p38 MAPK signalling, which in turn induces an upregulation of MMP activity to disrupt elastic fibre formation and degrade Fibronectin. However, the exact mechanisms by which FBN2 regulates p38 activity and p38 modulates $M m p$ expression remain to be determined. Transforming growth factor (TGF)- $\beta$ is abnormally activated in Fbn2-null osteoblasts [77]. Interestingly, TGF- $\beta$ inhibition has been reported to decrease p38 phosphorylation in smooth muscle cells [78]. It is possible that absence of FBN2 function activates TGF- $\beta$ signalling, thereby increasing p38 phosphorylation. Several targets of p38 MAPK have been reported to regulate $M m p 2$ and $M m p 9$ expression at the transcriptional level [66], and thus an increase in p38 phosphorylation might lead to the activation of specific transcription factor(s) whose target genes include $M m p 2$ and $M m p 9$.

Fibrillin microfibrils have been found within the matrix of human cartilage [79], and FBN2 mutations cause crumpled ears in patients $[80,81]$, which may be due to auricular cartilage defects. Our work presents evidence that Fibrillin microfibrils are required for cartilage formation possibly by promoting chondrocyte differentiation. Notably, FBN2 levels appear to be significantly decreased in the tracheal cartilage of tracheomalacia patients, suggesting that Fibrillin microfibrils are essential for cartilage formation also in humans. It will be interesting to investigate the underlying molecular mechanisms.

Mutations in FBN2 have been associated with congenital contractural arachnodactyly [80-83] and vascular disease in humans [84] as well as endocardial morphogenesis defects in zebrafish [85]. Our Fbn2 point mutant in mouse exhibits tracheal stenosis possibly caused by cartilage differentiation defects, providing a new model to study the aetiology of this disease, and develop therapeutic approaches.

Acknowledgements: We thank Francesco Ramirez (Icahn School of Medicine at Mount Sinai, New York, NY, USA) for providing Fbn2 null mice, Radhan Ramadass and Yu Hsuan Carol Yang for imaging assistance, Rubén Marín-Juez, Paolo Panza, Konstantinos Gkatzis, Srinath Ramkumar, Khrievono Kikhi, Jason Kuan Han Lai, Sharon Meaney-Gardian and Nana Fukuda (Max Planck Institute for Heart and Lung Research, Bad Nauheim, Germany) for discussion or assistance.

Author contributions: W. Yin and D.Y.R. Stainier conceived the project, designed experiments and analysed data; W. Yin, H-T. Kim, F. Gunawan, R. Li, B. Grohmann, C. Buettner, D. Sinner and S. Offermanns contributed to experiments and data analysis; S. Wang performed Western blot and trachea physiology experiments and data analysis; G. Sengle contributed to FBN2 antibody generation; W. Yin and D.Y.R. Stainier wrote the manuscript. All authors commented on the manuscript. 
Conflict of interest: None declared.

Support statement: Funding for this study was provided by the Deutsche Forschungsgemeinschaft (DFG) to G. Sengle (SFB829/ B11, B12) and the Max Planck Society to S. Offermanns and D.Y.R. Stainier.

\section{References}

1 Hogan BL, Kolodziej PA. Organogenesis: molecular mechanisms of tubulogenesis. Nat Rev Genet 2002; 3: 513-523.

2 Iruela-Arispe ML, Beitel GJ. Tubulogenesis. Development 2013; 140: 2851-2855.

3 Knot HJ, Nelson MT. Regulation of arterial diameter and wall $\left[\mathrm{Ca}^{2+}\right]$ in cerebral arteries of rat by membrane potential and intravascular pressure. J Physiol 1998; 508: 199-209.

4 Shyer AE, Tallinen T, Nerurkar NL, et al. Villification: how the gut gets its villi. Science 2013; 342: 212-218.

5 Kim HY, Pang MF, Varner VD, et al. Localized smooth muscle differentiation is essential for epithelial bifurcation during branching morphogenesis of the mammalian lung. Dev Cell 2015; 34: 719-726.

6 Hines EA, Jones MK, Verheyden JM, et al. Establishment of smooth muscle and cartilage juxtaposition in the developing mouse upper airways. Proc Natl Acad Sci USA 2013; 110: 19444-19449.

7 Domyan ET, Sun X. Patterning and plasticity in development of the respiratory lineage. Dev Dyn 2011; 240: 477-485.

8 Elluru RG, Whitsett JA. Potential role of Sox9 in patterning tracheal cartilage ring formation in an embryonic mouse model. Arch Otolaryngol Head Neck Surg 2004; 130: 732-736.

9 Snowball J, Ambalavanan M, Whitsett J, et al. Endodermal Wnt signaling is required for tracheal cartilage formation. Dev Biol 2015; 405: 56-70.

10 Yin W, Kim HT, Wang S, et al. The potassium channel KCNJ13 is essential for smooth muscle cytoskeletal organization during mouse tracheal tubulogenesis. Nat Commun 2018; 9: 2815.

11 Miller LA, Wert SE, Clark JC, et al. Role of Sonic hedgehog in patterning of tracheal-bronchial cartilage and the peripheral lung. Dev Dyn 2004; 231: 57-71.

12 Rawlins EL, Ostrowski LE, Randell SH, et al. Lung development and repair: contribution of the ciliated lineage. Proc Natl Acad Sci USA 2007; 104: 410-417.

13 Rawlins EL, Clark CP, Xue Y, et al. The Id $2^{+}$distal tip lung epithelium contains individual multipotent embryonic progenitor cells. Development 2009; 136: 3741-3745.

14 Rock JR, Hogan BL. Epithelial progenitor cells in lung development, maintenance, repair, and disease. Annu Rev Cell Dev Biol 2011; 27: 493-512.

15 Rackley CR, Stripp BR. Building and maintaining the epithelium of the lung. J Clin Invest 2012; 122: 2724-2730.

16 Landing BH, Dixon LG. Congenital malformations and genetic disorders of the respiratory tract (larynx, trachea, bronchi, and lungs). Am Rev Respir Dis 1979; 120: 151-185.

17 Fraga JC, Jennings RW, Kim PC. Pediatric tracheomalacia. Semin Pediatr Surg 2016; 25: 156-164.

18 Lubarsky B, Krasnow MA. Tube morphogenesis: making and shaping biological tubes. Cell 2003; 112: 19-28.

19 Andrew DJ, Ewald AJ. Morphogenesis of epithelial tubes: insights into tube formation, elongation, and elaboration. Dev Biol 2010; 341: 34-55.

20 Sala FG, Del Moral PM, Tiozzo C, et al. FGF10 controls the patterning of the tracheal cartilage rings via Shh Development 2011; 138: 273-282.

21 Gerhardt B, Leesman L, Burra K, et al. Notum attenuates Wnt/ $\beta$-catenin signaling to promote tracheal cartilage patterning. Dev Biol 2018; 436: 14-27.

22 Yu W, Datta A, Leroy P, et al. $\beta 1$-integrin orients epithelial polarity via Rac1 and laminin. Mol Biol Cell 2005; 16: 433-445.

23 Bryant DM, Roignot J, Datta A, et al. A molecular switch for the orientation of epithelial cell polarization. Dev Cell 2014; 31: 171-187.

24 Wang S, Sekiguchi R, Daley WP, et al. Patterned cell and matrix dynamics in branching morphogenesis. J Cell Biol 2017; 216: 559-570.

25 Li Q, Zhang Y, Pluchon P, et al. Extracellular matrix scaffolding guides lumen elongation by inducing anisotropic intercellular mechanical tension. Nat Cell Biol 2016; 18: 311-318.

26 Kile BT, Hentges KE, Clark AT, et al. Functional genetic analysis of mouse chromosome 11. Nature 2003; 425: 81-86.

27 Arteaga-Solis E, Gayraud B, Lee SY, et al. Regulation of limb patterning by extracellular microfibrils. J Cell Biol 2001; 154: 275-281.

28 Charbonneau NL, Carlson EJ, Tufa S, et al. In vivo studies of mutant fibrillin-1 microfibrils. J Biol Chem 2010; 285: 24943-24955.

29 Rigueur D, Lyons KM. Whole-mount skeletal staining. Methods Mol Biol 2014; 1130: 113-121.

30 Gosset M, Berenbaum F, Thirion S, et al. Primary culture and phenotyping of murine chondrocytes. Nat Protoc 2008; 3: 1253-1260.

31 Komatsu Y, Kishigami S, Mishina Y. In situ hybridization methods for mouse whole mounts and tissue sections with and without additional $\beta$-galactosidase staining. Methods Mol Biol 2014; 1092: 1-15.

32 Pilecki B, Holm AT, Schlosser A, et al. Characterization of microfibrillar-associated protein 4 (MFAP4) as a tropoelastin- and fibrillin-binding protein involved in elastic fiber formation. J Biol Chem 2016; 291: 1103-1114.

33 Del Moral PM, Warburton D. Explant culture of mouse embryonic whole lung, isolated epithelium, or mesenchyme under chemically defined conditions as a system to evaluate the molecular mechanism of branching morphogenesis and cellular differentiation. Methods Mol Biol 2010; 633: 71-79.

34 Bi W, Deng JM, Zhang Z, et al. Sox9 is required for cartilage formation. Nat Genet 1999; 22: 85-89.

35 Yanagino T, Yuasa K, Nagahama M, et al. Transcriptional regulation of fibrillin-2 gene by E2F family members in chondrocyte differentiation. J Cell Biochem 2009; 106: 580-588.

36 Kishimoto K, Tamura M, Nishita M, et al. Synchronized mesenchymal cell polarization and differentiation shape the formation of the murine trachea and esophagus. Nat Commun 2018; 9: 2816. 
37 Li Y, Dudley AT. Noncanonical frizzled signaling regulates cell polarity of growth plate chondrocytes. Development 2009; 136: 1083-1092.

38 Nobes CD, Hall A. Rho GTPases control polarity, protrusion, and adhesion during cell movement. J Cell Biol 1999; 22: 1235-1244.

39 Kwon HB, Wang S, Helker CS, et al. In vivo modulation of endothelial polarization by Apelin receptor signalling. Nat Commun 2016; 7: 11805.

40 Hannezo E, Dong B, Recho P, et al. Cortical instability drives periodic supracellular actin pattern formation in epithelial tubes. Proc Natl Acad Sci USA 2015; 112: 8620-8625.

41 Billmyre KK, Hutson M, Klingensmith J. One shall become two: separation of the esophagus and trachea from the common foregut tube. Dev Dyn 2015; 244: 277-288.

42 Kablar B, Tajbakhsh S, Rudnicki MA. Transdifferentiation of esophageal smooth to skeletal muscle is myogenic bHLH factor-dependent. Development 2000; 127: 1627-1639.

43 Bunton TE, Biery NJ, Myers L, et al. Phenotypic alteration of vascular smooth muscle cells precedes elastolysis in a mouse model of Marfan syndrome. Circ Res 2001; 88: 37-43.

44 Carta L, Pereira L, Arteaga-Solis E, et al. Fibrillins 1 and 2 perform partially overlapping functions during aortic development. J Biol Chem 2006; 281: 8016-8023.

45 Kielty CM. Elastic fibres in health and disease. Expert Rev Mol Med 2006; 8: 1-23.

46 Lucero HA, Kagan HM. Lysyl oxidase: an oxidative enzyme and effector of cell function. Cell Mol Life Sci 2006; 63: 2304-2316.

47 Jensen SA, Robertson IB, Handford PA. Dissecting the fibrillin microfibril: structural insights into organization and function. Structure 2012; 20: 215-225.

48 Clarke AW, Wise SG, Cain SA, et al. Coacervation is promoted by molecular interactions between the PF2 segment of fibrillin-1 and the domain 4 region of tropoelastin. Biochemistry 2005; 44: 10271-10281.

49 Schwill S, Seppelt P, Grünhagen J, et al. The fibrillin-1 hypomorphic mgR/mgR murine model of Marfan syndrome shows severe elastolysis in all segments of the aorta. J Vasc Surg 2013; 57: 1628-1636.

50 Ju X, Ijaz T, Sun H, et al. IL-6 regulates extracellular matrix remodeling associated with aortic dilation in a fibrillin-1 hypomorphic mgR/mgR mouse model of severe Marfan syndrome. J Am Heart Assoc 2014; 3: e000476.

51 Hubmacher D, Wang LW, Mecham RP, et al. Adamtsl2 deletion results in bronchial fibrillin microfibril accumulation and bronchial epithelial dysplasia - a novel mouse model providing insights into geleophysic dysplasia. Dis Model Mech 2015; 8: 487-499.

52 Pierschbacher MD, Ruoslahti E. Cell attachment activity of fibronectin can be duplicated by small synthetic fragments of the molecule. Nature 1984; 309: 30-33.

53 Sabatier L, Chen D, Fagotto-Kaufmann C, et al. Fibrillin assembly requires fibronectin. Mol Biol Cell 2009; 20: 846-858.

54 Martins GG, Rifes P, Amândio R, et al. Dynamic 3D cell rearrangements guided by a fibronectin matrix underlie somitogenesis. PLoS One 2009; 4: e7429.

55 Vaz R, Martins GG, Thorsteinsdóttir S, et al. Fibronectin promotes migration, alignment and fusion in an in vitro myoblast cell model. Cell Tissue Res 2012; 348: 569-578.

56 Ray D, Osmundson EC, Kiyokawa H. Constitutive and UV-induced fibronectin degradation is a ubiquitination-dependent process controlled by $\beta$-TrCP. J Biol Chem 2006; 281: 23060-23065.

57 Li DY, Brooke B, Davis EC, et al. Elastin is an essential determinant of arterial morphogenesis. Nature 1998; 393: 276-280.

58 Wagenseil JE, Ciliberto $\mathrm{CH}$, Knutsen $\mathrm{RH}$, et al. Reduced vessel elasticity alters cardiovascular structure and function in newborn mice. Circ Res 2009; 104: 1217-1224.

59 Kenny HA, Kaur S, Coussens LM, et al. The initial steps of ovarian cancer cell metastasis are mediated by MMP-2 cleavage of vitronectin and fibronectin. J Clin Invest 2008; 118: 1367-1379.

60 Yabluchanskiy A, Ma Y, Iyer RP, et al. Matrix metalloproteinase-9: many shades of function in cardiovascular disease. Physiology 2013; 28: 391-403.

61 Oller J, Méndez-Barbero N, Ruiz EJ, et al. Nitric oxide mediates aortic disease in mice deficient in the metalloprotease Adamts1 and in a mouse model of Marfan syndrome. Nat Med 2017; 23: 200-212.

62 Kim HT, Yin W, Jin YJ, et al. Myh10 deficiency leads to defective extracellular matrix remodeling and pulmonary disease. Nat Commun 2018; 9: 4600.

63 Grobelny D, Poncz L, Galardy RE, et al. Inhibition of human skin fibroblast collagenase, thermolysin, and Pseudomonas aeruginosa elastase by peptide hydroxamic acids. Biochemistry 1992; 31: 7152-7154.

64 Skjøt-Arkil H, Clausen RE, Nguyen QH, et al. Measurement of MMP-9 and -12 degraded elastin (ELM) provides unique information on lung tissue degradation. BMC Pulm Med 2012; 12: 34.

65 Gill SE, Pape MC, Leco KJ, et al. Tissue inhibitor of metalloproteinases 3 regulates extracellular matrix-cell signaling during bronchiole branching morphogenesis. Dev Biol 2006; 298: 540-554.

66 Koul HK, Pal M, Koul S. Role of p38 MAP kinase signal transduction in solid tumors. Genes Cancer 2013; 4 342-359.

67 Song $\mathrm{H}$, Ki SH, Kim SG, et al. Activating transcription factor 2 mediates matrix metalloproteinase-2 transcriptional activation induced by p38 in breast epithelial cells. Cancer Res 2006; 66: 10487-10496.

68 Kumar B, Koul S, Petersen J, et al. p38 mitogen-activated protein kinase-driven MAPKAPK2 regulates invasion of bladder cancer by modulation of MMP-2 and MMP-9 activity. Cancer Res 2010; 70: 832-841.

69 Cuenda A, Rouse J, Doza YN, et al. SB 203580 is a specific inhibitor of a MAP kinase homologue which is stimulated by cellular stresses and interleukin-1. FEBS Lett 1995; 364: 229-233.

70 Turcatel G, Rubin N, Menke DB, et al. Lung mesenchymal expression of Sox9 plays a critical role in tracheal development. BMC Biol 2013; 11: 117.

71 Bonvin E, Le Rouzic P, Bernaudin JF, et al. Congenital tracheal malformation in cystic fibrosis transmembrane conductance regulator-deficient mice. J Physiol 2008; 586: 3231-3243.

72 Wallace HL, Southern KW, Connell MG, et al. Abnormal tracheal smooth muscle function in the CF mouse. Physiol Rep 2013; 1: e00138.

73 Lin SS, Tzeng BH, Lee KR, et al. Cav3.2 T-type calcium channel is required for the NFAT-dependent Sox9 expression in tracheal cartilage. Proc Natl Acad Sci USA 2014; 111: E1990-E1998. 
74 Carta L, Smaldone S, Zilberberg L, et al. p38 MAPK is an early determinant of promiscuous Smad2/3 signaling in the aortas of fibrillin-1 (Fbn1)-null mice. J Biol Chem 2009; 284: 5630-5636.

75 Xiong W, Knispel RA, Dietz HC, et al. Doxycycline delays aneurysm rupture in a mouse model of Marfan syndrome. J Vasc Surg 2008; 47: 166-172.

76 Kinsey R, Williamson MR, Chaudhry S, et al. Fibrillin-1 microfibril deposition is dependent on fibronectin assembly. J Cell Sci 2008; 121: 2696-2704.

77 Nistala H, Lee-Arteaga S, Smaldone S, et al. Fibrillin-1 and -2 differentially modulate endogenous TGF- $\beta$ and BMP bioavailability during bone formation. J Cell Biol 2010; 190: 1107-1121.

78 Granata A, Serrano F, Bernard WG, et al. An iPSC-derived vascular model of Marfan syndrome identifies key mediators of smooth muscle cell death. Nat Genet 2017; 49: 97-109.

79 Keene DR, Jordan CD, Reinhardt DP, et al. Fibrillin-1 in human cartilage: developmental expression and formation of special banded fibers. J Histochem Cytochem 1997; 45: 1069-1082.

80 Mehar V, Yadav D, Kumar R, et al. Congenital contractural arachnodactyly due to a novel splice site mutation in the FBN2 gene. J Pediatr Genet 2014; 3: 163-166.

81 Lavillaureix A, Heide S, Chantot-Bastaraud S, et al. Mosaic intragenic deletion of FBN2 and severe congenital contractural arachnodactyly. Clin Genet 2017; 92: 556-558.

82 Putnam EA, Zhang H, Ramirez F, et al. Fibrillin-2 (FBN2) mutations result in the Marfan-like disorder, congenital contractural arachnodactyly. Nat Genet 1995; 11: 456-458.

83 Gupta PA, Wallis DD, Chin TO, et al. FBN2 mutation associated with manifestations of Marfan syndrome and congenital contractural arachnodactyly. J Med Genet 2004; 41: e56.

84 Kunnas T, Solakivi T, Nikkari ST, et al. Gene polymorphisms of fibronectin rs2289202 and fibrillin 2 rs331069 associate with vascular disease, the TAMRISK study. Biomed Rep 2018; 8: 65-68.

85 Mellman K, Huisken J, Dinsmore C, et al. Fibrillin-2b regulates endocardial morphogenesis in zebrafish. Dev Biol 2012; 372: 111-119. 